\title{
Local solution of Cauchy problem for nonlinear hyperbolic systems in Gevrey classes
}

\author{
By Kunihiko KajitanI
}

(Received January 4, 1983)

\section{Introduction}

The Cauchy problem for nonlinear hyperbolic equations in Gevrey classes was studies by Leray-Ohya [7] (c.f. [8]). They assume that the characteristics are of constant multiplicity or smooth. In this paper we shall remove this restriction.

We consider the following equations for the unknowns $u(x)=\left(u_{1}(x), \cdots\right.$, $\left.u_{N}(x)\right), x=\left(x_{0}, x_{1}, \cdots, x_{n}\right)=\left(x_{0}, x^{\prime}\right) \in R^{n+1}$,

$$
F_{i}\left(x, D^{M_{i}} u(x)\right)=0 \text { in } \Omega, \quad i=1, \cdots, N,
$$

where $\Omega$ is a neighborhood of 0 in $R^{n+1}$ and

$$
\begin{aligned}
& D^{M_{i}} u(x)=\left\{D^{M_{i 1}} u_{1}(x), \cdots, D^{M_{i N}} u_{N}(x)\right\} \\
& D^{M_{i j}} u_{j}(x)=\left\{\left(\partial / \partial x_{0}\right)^{\alpha_{0}}\left(\partial / \partial x_{1}\right)^{\alpha_{1}} \cdots\left(\partial / \partial x_{n}\right)^{\alpha_{n}} u_{j}(x) ; \alpha=\left(\alpha_{0}, \alpha_{1}, \cdots, \alpha_{n}\right) \in M_{i j}\right\}
\end{aligned}
$$

and $M_{i j}$ is a finite set of non negative multi indices.

We assume that $\left\{F_{i}\right\}$ is a Leray-Volevich system of order $m$, that is, there exist non negative integers $n_{1}, \cdots, n_{N}$ such that for $\alpha \in M_{i j}$,

$$
|\alpha|=\alpha_{0}+\alpha_{1}+\cdots+\alpha_{n} \leqslant m+n_{j}-n_{i}, \quad i, j=1, \cdots, N .
$$

Then we can prescribe the following Cauchy data to the equations (0.1),

$$
\left(\partial / \partial x_{0}\right)^{j} u_{i}\left(0, x^{\prime}\right)=\varphi_{j i}\left(x^{\prime}\right), \quad j=0, \cdots, m-1, i=1, \cdots, N .
$$

We introduce coordinate variables

$$
\begin{aligned}
& y_{i j}=\left(y_{\alpha} ; \alpha \in M_{i j}\right) \text { in } R^{r_{i j}}, \quad i, j=1, \cdots, N, \\
& y_{i}=\left(y_{i j} ; j=1, \cdots, N\right) \text { in } R^{r_{i}}, \quad i=1, \cdots N, \\
& y=\left(y_{i} ; i=1, \cdots, N\right) \text { in } R^{r},
\end{aligned}
$$

where $r_{i j}$ is the number of the elements of $M_{i j}, r_{i}=r_{i 1}+\cdots+r_{i N}$ and $r=$ $r_{1}+\cdots+r_{N}$.

We assume that $F_{i}(x, y), i=1, \cdots, N$ are in Gevrey class $s$ in $x$ and 
analytic in $y \in V\left(V\right.$ is a compact set in $\left.R^{r}\right)$, that is, there are positive numbers $C$ and $A$ such that

$$
\left|D_{x}^{\alpha} D_{y}^{\beta} F_{i}(x, y)\right| \leqslant C A^{|\alpha|+|\beta|}|\alpha| !^{s}|\beta| !, x \text { in } \Omega, \quad y \text { in } V,
$$

for $\alpha \in N^{n+1}, \beta \in N^{r}$.

We define the characteristic matrix for $\left\{F_{i}\right\}$ as follows

$$
p_{i j}(x, y, \xi)=\sum_{\substack{|\alpha|=m+n_{j}-n_{i} \\ \alpha \in M_{i j}}}\left(\partial / \partial y_{\alpha}\right) F_{i}(x, y) \xi^{\alpha}, \quad i, j=1, \cdots, N,
$$

which is a polynomial in $\xi$ of degree $m+n_{j}-n_{i}$. We call the determinant of $\left\{p_{i j}(x, y, \xi)\right\}$ characteristic polynomial and denote it by $p(x, y, \xi)$. We say that a system $\left\{F_{i}\right\}$ is hyperbolic in $\Omega \times V$ with respect to $\xi_{0}$, if the characteristic polynomial $p\left(x, y, \xi_{0}-\sqrt{-1} \lambda, \xi^{\prime}\right)$ does not vanish for $(x, y, \xi, \lambda)$ in $\Omega \times V \times R^{n+1} \times R^{1} \backslash 0$ and $p(x, y, 1,0)=1$. Then $p\left(x, y, \xi_{0}, \xi^{\prime}\right)$ has only real roots $\lambda_{j}\left(x, y, \xi^{\prime}\right)(j=1, \cdots, m N)$ with respect to $\xi_{0}$ for $\left(x, y, \xi^{\prime}\right)$ in $G \times V \times R^{n} \backslash 0$. Assume that the multiplicities of the roots $\lambda_{j}\left(x, y, \xi^{\prime}\right)$ do not exceed $\nu$ for any $\left(x, y, \xi^{\prime}\right) \in G \times V \times R^{n} \backslash 0$.

For $K$ a closed set in $R^{n}$, we denote by $\gamma_{A}^{(s)}(K)$ the class of all functions $u\left(x^{\prime}\right)$ satisfying

$$
\left|D^{\alpha} u\left(x^{\prime}\right)\right| \leqslant C A^{|\alpha|}|\alpha| !^{s},
$$

for $x^{\prime} \in K$ and $\alpha \in N^{n}$, and by $\gamma_{A}^{k(s)}([0, T] \times K)$ the class of all $k$ times continuously differentiable function of $x_{0}$ in $\gamma_{A}^{(s)}(K)$. We denote $\gamma^{k(s)}=\bigcup_{A>0} \gamma_{A}^{k(s)}$ and $\gamma^{\infty(s)}=\bigcap_{k \geq 0} \gamma^{k(s)}$

We consider the Cauchy problem $(0,1)$ with intial data $(0.3)$. Then we obtain following theorems,

Theorem 0.1. Assume that $\left\{F_{i}(x, y)\right\}$ is a hyperbolic Leray-Volevich system of order $m$, satisfying (0.4), and that the multiplicities of the roots of it's characteristic polynomial do not exceed $\nu$. If $1<s<\nu(\nu-1)^{-1}$, then for any initial data $\left\{\varphi_{j i}\left(x^{\prime}\right)\right\}$ in $\gamma^{(s)}(K)$, there exist $T>0$ and $K_{0} \subset K$ such that the Cauchy problem (0.1) and (0.3) has a solution $\left\{u_{i}(x)\right\}$ in $\gamma^{\infty(s)}\left([0, T] \times K_{0}\right)$.

THEOREM 0.2. Assume that the conditions of Theorem 0.1 are valid. Let $\left\{\varphi_{j i}\left(x^{\prime}\right)\right\}$ and $\left\{\varphi_{j i}\left(x^{\prime}\right)\right\}$ be in $\gamma^{(s)}(K)$ such that $\varphi_{j i}\left(x^{\prime}\right)=\phi_{j i}\left(x^{\prime}\right)$ for $x^{\prime} \in K_{0}$ $\subset K$. Let $\left\{u_{i}\right\}$ and $\left\{v_{i}\right\}$ be a solution of $(0.1)$ with initial data $\left\{\varphi_{j i}\right\}$ and $\left\{\phi_{j i}\right\}$ respectively. Then there exists a positive number $\lambda_{0}$ such that

$$
\left\{u_{i}(x)\right\}=\left\{v_{i}(x)\right\} \text { for } x \in\left\{x \in R^{n+1} ; 0 \leqslant x_{0} \leqslant \lambda_{0}\left|x^{\prime}-y^{\prime}\right|, y^{\prime} \in K_{0}\right\} \text {. }
$$

Recently, in the linear case, the above theorems have proved by Bronshtein [2]. Kajitani [4] and Nishitani [6] have given another proof. To 
prove Theorem 0.1 and 0.2 we shall derive an energy estimate for the linearized equations of $(0.1)$ by methods in [4] and apply Schauder's fixed point theorem.

\section{$\S 1$. Preliminaries}

We introduce the following notation; $x=\left(x_{0}, x_{1}, \cdots, x_{n}\right)=\left(x_{0}, x^{\prime}\right)$ in $R^{n+1}$ and $\xi=\left(\xi_{0}, \xi^{\prime}\right)$ is dual variable with a inner product $x \xi=\sum_{i} x_{i} \xi_{i}, D=\left(D_{0}\right.$, $\left.D_{1}, \cdots, D_{n}\right), D_{j}=-\sqrt{-1} \partial / \partial x_{j} \alpha=\left(\alpha_{0}, \alpha_{1}, \cdots, \alpha_{n}\right)=\left(\alpha_{0}, \alpha^{\prime}\right)$ in $N^{n+1},|\alpha|=\Sigma \alpha_{i},\langle\xi\rangle_{h}^{2}$ $=h^{2}+\xi_{0}^{2}+\xi_{1}^{2}+\cdots+\xi_{n}^{2},\left\langle\xi^{\prime}\right\rangle_{h}^{2}=h^{2}+\xi_{1}^{2}+\cdots, \xi_{n}^{2},\langle D\rangle_{h}^{2}=h^{2}-\Delta_{x},\left\langle D^{\prime}\right\rangle_{h}^{2}=h^{2}-\Delta_{x^{\prime}}$, $(h>0)$. We denote by $W_{l, q, h}$, where $l, q$ in $R^{1}$ and $h>0$, the class of functions $u(x)$ such that $\langle D\rangle_{h}^{l}\left\langle D^{\prime}\right\rangle_{h}^{q} u$ in $L^{2}\left(R^{n+1}\right)$. We put $\Lambda=\Lambda\left(x_{0}, T, h, \kappa, D^{\prime}\right)$ $=\left(T-x_{0}\right)\left(h+\left\langle D^{\prime}\right\rangle_{h}^{k}\right)$, where $T \in R^{1}, h>0$ and $\kappa=s^{-1}$. We define $e^{A} u(x)$,

$$
e^{\Lambda} u(x)=e^{\Lambda\left(x_{0}, D^{\prime}\right)} u(x)=\int e^{\left[i x^{\prime} \xi^{\prime}+\Lambda\left(x_{0}, T, h, \kappa, \xi^{\prime}\right)\right]} \tilde{u}\left(x_{0}, \xi^{\prime}\right) d \xi^{\prime},
$$

where $d \xi^{\prime}=(2 \pi)^{-n} d \xi^{\prime}$ and $\tilde{u}\left(x_{0}, \xi^{\prime}\right)$ stands for a Fourier transform of $u$ with respect to $x^{\prime}$. Denote by $W_{i, q, h}^{(\Lambda)}$ all functions $u(x)$ such that $e^{\Lambda} u$ in $W_{l, q, h}$. When $l$ is a non negative integer, for $\Omega_{T_{0}}=\left[0, T_{0}\right] \times R^{n},\left(T_{0}>0\right)$, we define $W_{l, q, h}\left(\Omega_{T_{0}}\right)$, all functions $u(x)$ such that $D_{0}^{j}\left\langle D^{\prime}\right\rangle_{h}^{l-j+q} u(j=0, \cdots, l)$, in $L^{2}\left(\Omega_{T_{0}}\right)$ and also define $W_{i, q, h}^{(1)}\left(\Omega_{T_{0}}\right)$ analogously.

LemMA 1.1. Let $f$ and $g$ be in $W_{l, q, h}^{(1)}$, where $\Lambda=\left(T-x_{0}\right)\left(h+\left\langle D^{\prime}\right\rangle_{h}^{k}\right)$, $T \geq 0$ and $0<\kappa<1$. Then if $(n+2), q \geq 0$, and supp $f \subset\left\{x_{0} \leqslant \frac{T}{2}\right\}$, then the product $f \cdot g$ is also in $W_{l, q, h}^{(1)}$ and satisfies

$$
\|f \cdot g\|_{W_{l, q, h}^{(A)}} \leqslant C_{l}\|f\|_{W_{l, q, h}^{(\lambda)}}\|g\|_{W_{l, q, h}^{(A)}},
$$

where $C_{l}$ is independent of $h$.

Proof. We have

$$
\begin{aligned}
& \|f g\|_{W_{l, q, h}^{(1)}}^{2}=\sum_{|\beta|+j \leqslant l}\left\|e^{\Lambda} h^{l-j} D^{\beta}(f g)\right\|_{W_{0}, q, h}^{2} \\
& \leqslant C \sum_{\substack{|\beta|+j \leqslant l \\
\beta^{\prime}+\beta^{\prime \prime}=\beta}}\left\|e^{4} h^{l-j} D^{\beta^{\prime}} f D^{\beta^{\prime \prime}} g\right\|_{W_{0}, q, h}^{2} \\
& \leqslant C h^{2 l} \sum_{\beta^{\prime}+\beta^{\prime \prime}=\beta}\left\|e^{4\left(\xi^{\prime}\right)}\left\langle\xi^{\prime}\right\rangle=\int \tilde{f}_{\beta^{\prime}}\left(x_{0}, \xi^{\prime}-\eta^{\prime}\right) \tilde{g}_{\beta^{\prime \prime}}\left(x_{0}, \eta^{\prime}\right) d \eta^{\prime}\right\|_{L^{2}}^{2} \\
& \leqslant C h^{2 l} \sum \| K\left(x_{0}, \xi^{\prime}, h\right) \int e^{\Lambda\left(\xi^{\prime}-\eta^{\prime}\right)}\left\langle\xi^{\prime}-\eta^{\prime}\right\rangle_{h}^{q}{\tilde{\beta^{\prime}}}_{\beta^{\prime}}\left(x_{0}, \xi^{\prime}-\eta^{\prime}\right) \\
& \times\left. e^{\Lambda\left(\eta^{\prime}\right)}\left\langle\eta^{\prime}\right\rangle_{h}^{q} \tilde{g}_{\beta^{\prime \prime}}\left(x_{0}, \eta^{\prime}\right) d \eta\right|_{L^{2}} ^{2}
\end{aligned}
$$

where $\tilde{f}_{\beta^{\prime}}\left(x_{0}, \xi^{\prime}\right)$ and $\tilde{g}_{\beta^{\prime \prime}}$ are Fourier transforms of $D^{\beta^{\prime}} f$ and $D^{\beta^{\prime \prime}} g$ with 
respect to $x^{\prime}$ and

$$
K\left(x_{0}, \xi^{\prime}, h\right)=\inf _{\eta^{\prime} \in R^{n}} e^{\Lambda\left(\xi^{\prime}\right)-\Lambda\left(\xi^{\prime}-\eta^{\prime}\right)-\Lambda\left(\eta^{\prime}\right)}\left\{\left\langle\xi^{\prime}\right\rangle_{h}\left\langle\xi^{\prime}-\eta^{\prime}\right\rangle_{h}^{-1}\left\langle\eta^{\prime}\right\rangle_{h}^{-1}\right\}^{q} .
$$

Noting that $\Lambda\left(\xi^{\prime}\right)-\Lambda\left(\xi^{\prime}-\eta^{\prime}\right)-\Lambda\left(\eta^{\prime}\right)=\left(T-x_{0}\right)\left(\left\langle\xi^{\prime}\right\rangle_{h}^{\kappa}-\left\langle\xi^{\prime}-\eta^{\prime}\right\rangle_{h}^{\boldsymbol{c}}-\left\langle\eta^{\prime}\right\rangle_{h}^{\boldsymbol{c}}\right) \leqslant-h T / 2$ for $x_{0} \leqslant T / 2$ and $\left(\left\langle\xi^{\prime}\right\rangle_{h}\left\langle\xi^{\prime}-\eta^{\prime}\right\rangle_{h}^{-1}\left\langle\eta^{\prime}\right\rangle_{h}^{-1}\right)^{q} \leqslant 1$ for $q \geqslant 0$, we have

$$
K\left(x_{0}, \xi^{\prime}, h\right) \leqslant e^{-h T / 2}, \quad x_{0} \leqslant T / 2 .
$$

Hence we obtain by virtue of Hausdorff-Yong's inequality,

$$
\begin{aligned}
& \|f g\|_{W_{l, q, h}^{(A)}}^{2} \leqslant C e^{-T h} h^{2}\left\{\sum_{\left|\beta^{\prime}\right| \geqslant \frac{l}{2}}\left\|\left.e^{\Lambda\left(\xi^{\prime}\right)}\left\langle\xi^{\prime}\right\rangle{ }_{h}^{q} \tilde{f}_{\beta^{\prime}}\right|_{L^{2}} ^{2}\right\| e^{\Lambda\left(\xi^{\prime}\right)}\left\langle\xi^{\prime}\right\rangle_{h}^{q} \tilde{g}_{\beta^{\prime}} \|_{L^{1}}^{2}\right. \\
& \left.+\sum_{\left|\beta^{\prime}\right|<\frac{l}{2}}\left\|e^{\Lambda\left(\xi^{\prime}\right)}\left\langle\xi^{\prime}\right\rangle{ }_{h}^{q} \tilde{f}_{\beta^{\prime}}\right\|_{L^{1}}^{2}\left\|e^{\Lambda\left(\xi^{\prime}\right)}\left\langle\xi^{\prime}\right\rangle_{h}^{q} \tilde{g}_{\beta^{\prime}}\right\|_{L^{2}}^{2}\right\} \\
& \leqslant C\left\{\|f\|_{w_{l, q, h}^{(1)}}\|g\|_{w_{l}^{(j)+[n / 2]+1, q, h}}\right. \\
& \left.+\|f\|_{W_{l / 2+[n / 2]+1, q, h}^{(1)}}\|g\|_{W_{l, q, h}^{(1)}}\right\} \\
& \leqslant C\|f\|_{W_{l, q, h}^{(A)}}\|g\|_{W_{l, q, h}^{(A)}}, \quad \text { for } \quad l \geqslant n+2 \text {. }
\end{aligned}
$$

LEMMA 1.2. Let $f$ be in $W_{l, q, h}^{(1)}, g$ in $W_{m, q, h}^{(1)}$ and supp $f \subset\left\{x_{0} \leqslant T\right\}$. Then if $l$ is sufficiently large and $q \geqslant 0$, the product $f \cdot g$ is in $W_{m, q, h}^{(1)}$ and satisfies

$$
\|f \cdot g\|_{W_{m, q, h}^{(A)}} \leqslant C\|f\|_{W_{l, q, h}^{(A)}}\|g\|_{W_{m, q, h}^{(1)}},
$$

where $\Lambda=\left(T-x_{0}\right)\left(h+\left\langle D^{\prime}\right\rangle_{h}^{k}\right), T \geqslant 0,0<\kappa<1$, and $C$ is independent of $h$.

This lemma follows from Proposition 2.7 in $\S 2$.

LEMMA 1.3. Let $u(x)$ be in $\gamma_{A_{0}}^{l(s)}$ and of a compact support in $\left\{x \in R^{n+1}\right.$; $\left.x_{0} \geqslant 0\right\}$, where $l$ a positive integer. Then there exists $T>0$ such that for any $q \in R^{1}$ and $h>0, u(x)$ is in $W_{l, q, h}^{(1)}$, where $\Lambda=\left(T-x_{0}\right)\left(h+\left\langle D^{\prime}\right\rangle_{h}^{k}\right), \kappa=s^{-1}$.

Proof. Since $u$ is in $\gamma_{A_{0}}^{l(s)}, u$ satisfies

$$
\left|D^{\alpha} u(x)\right| \leqslant C A_{0}^{\left|\alpha^{\prime \prime}\right|}\left|\alpha^{\prime}\right| ! s
$$

for $\alpha=\left(\alpha_{0}, \alpha^{\prime}\right)$ in $N^{n+1}, \alpha_{0} \leqslant l$. Hence

$$
\begin{aligned}
\left|\xi^{\alpha^{\prime}} D_{0}^{\alpha} \tilde{u}\left(x_{0}, \xi^{\prime}\right)\right| & =\left|\int e^{-i x^{\prime} \xi^{\prime}} D^{\alpha} u(x) d x^{\prime}\right| \\
& \leqslant C A_{0}^{\left|\alpha^{\prime}\right|}\left|\alpha^{\prime}\right| !^{!},
\end{aligned}
$$

for $\alpha=\left(\alpha_{0}, \alpha^{\prime}\right)$ in $N^{n+1}, \alpha_{0} \leqslant l$, which implies

$$
\left|D_{0}^{\alpha_{0}} \tilde{u}\left(x_{0}, \xi^{\prime}\right)\right| \leqslant C_{l}\left(A_{0}\left\langle\xi^{\prime}\right\rangle^{-1}\right)^{M} M !^{s}, \quad \alpha_{0} \leqslant l
$$


for any positive integer $M$, where $\left\langle\xi^{\prime}\right\rangle=\left(1+\left|\xi^{\prime}\right|^{2}\right)^{1 / 2}$. :Therefore,

$$
\left|D_{0}^{\alpha_{0}} u\left(x_{0}, \xi^{\prime}\right)\right| \leqslant \inf _{M \in N} C\left(A_{0}\left\langle\xi^{\prime}\right\rangle^{-1}\right)^{M} M !^{s} \leqslant C_{1} e^{-\left(\left\langle A_{0}^{-1}\left\langle\xi^{\prime}\right\rangle\right)^{1 / s} / 2\right.}
$$

and

$$
\begin{aligned}
& e^{\left(T-x_{0}\right)\left(h+\left\langle\xi^{\prime}\right\rangle_{h}^{\kappa}\right)}\left\langle\xi^{\prime}\right\rangle_{h}^{l+q-\alpha_{0}}\left|D_{0}^{\alpha_{0}} \tilde{u}\left(x_{0}, \xi^{\prime}\right)\right| \\
& \quad \leqslant C_{2}(T, h) e^{\left(T-x_{0}\right)\left\langle\xi^{\prime}\right\rangle}\left\langle\xi^{\prime}\right\rangle^{l+q-\alpha_{0}}\left|D_{0}^{\alpha_{0}} \tilde{u}\left(x_{0}, \xi^{\prime}\right)\right| \\
& \quad \leqslant C_{3}(T, h) e^{-\left\{\left(A_{0}^{-1}\left\langle\xi^{\prime}\right\rangle\right)^{1 / s_{3}} / 4\right.},
\end{aligned}
$$

if $T-x_{0} \leqslant A_{0}^{-1 / s} / 4$ and $x_{0} \geqslant 0$, which implies our conclusion.

Next we mention the properties of hyperbolic polynomials.

Lemma 1.4. Let $p(z, \xi)=\sum_{|\alpha|=m} a_{\alpha}(z) \xi^{\alpha}$ be a hyperbolic polynomial with respect to $\xi_{0}$, which coefficients $a_{\alpha}(z)$ are constant in the compliment of a compact set in $R^{d}$ and which multiplicity of roots is at most $\nu$. Then the following properties hold,

(i) $\left|p\left(z, \xi_{1}\right)^{-1}\right| \leqslant C\langle\xi\rangle_{h}^{-m}\left\langle\xi^{\prime}\right\rangle_{h}^{z}, \quad(z, \xi) \in R^{d+n+1}$,

(ii) $\left|p^{(\alpha)}\left(z, \xi_{1}\right) p\left(z, \xi_{A}\right)^{-1}\right| \leqslant C\left\langle\xi^{\prime}\right\rangle_{h}^{-\kappa}, \quad(z, \xi) \in R^{d+n+1},|\alpha|=1$,

(iii) $\left|p_{(\alpha)}\left(z, \xi_{A}\right) p\left(z, \xi_{A}\right)^{-1}\right| \leqslant C\left\langle\xi^{\prime}\right\rangle_{h}^{1-\kappa}, \quad(z, \xi) \in R^{d+n+1},|\alpha|=1 ，$

where $C$ is independent of $h$ and $\xi_{A}=\left(\xi_{0}-i\left(h+\left\langle\xi^{\prime}\right\rangle_{h}^{k}, \xi^{\prime}\right), \tau=\nu(1-\kappa)\right.$.

The proof of (ii) and (iii) follows from Bronshtein [1].

\section{$\S$ 3. Pseudo-differential operators in Gevrey class}

We introduce a class of pseudo-differential operators in Gevrey classes.

Definition 2.1. Denote by $S_{\rho, \dot{\delta}, h}^{(p)}$, a set of all functions $a\left(x^{\prime}, \xi^{\prime}\right)$ in $C^{\infty}\left(R^{2 n}\right)$ such that

$$
|a|_{l}^{(p)}=\sup _{\substack{\left(x^{\prime}, \xi^{\prime}\right) \in \mathcal{R}^{2 n} \\|\alpha+\beta| \leq l}} \frac{\left|a^{(\alpha)}(\beta)\left(x^{\prime}, \xi^{\prime}\right)\right|}{\left\langle\xi^{\prime}\right\rangle h-p|\alpha|+\delta|\beta|}<\infty
$$

for each $l \in N$, where $p \in R^{1}$, and $0 \leqslant \delta \leq \rho \leq 1$.

Definition 2.2. Denote by $S_{\rho, \delta, h}^{(m, p)}$ a set of all functions $a(x, \xi) \in C^{\infty}\left(R^{2(n+1)}\right)$ such that

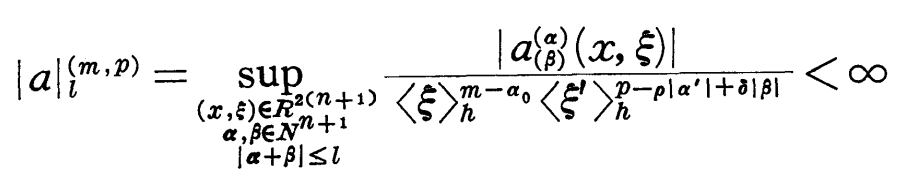


for each $l \in N$, where $m, q \in R^{1}, 0 \leqslant \delta \leqslant \rho \leq 1$.

We define $\mathscr{A}_{s, A, h}^{(p)}$ all functions $a\left(x^{\prime}, \xi^{\prime}\right)$ satisfying

$$
|a|_{s, A, l}^{(p)}=\sup _{\substack{\gamma \in N^{n} \\|a+\beta| \leq l \\\left(x^{\prime}, \xi^{\prime}\right) \in R^{2 n}}} \frac{\left|a^{(\alpha)}(\beta+\gamma)\left(x^{\prime}, \xi^{\prime}\right)\right|}{A^{|r|}|\gamma| !^{s}\left\langle\xi^{\prime}\right\rangle_{h}^{p-|\alpha|}}<\infty,
$$

for each $l \in N$, and also define by $C_{0}^{\infty}\left([-T, T] ; \mathscr{A}_{s, A, h}^{(p)}\right)$ all functions a $\left(x_{0}, x^{\prime}, \xi^{\prime}\right)$ satisfying

$$
[a]_{s, A, l}^{(p)}=\sup _{\substack{0 \leq i \leq l \\\left|x_{0}\right| \leqslant T}}\left|\left(\frac{\partial}{\partial x_{0}}\right)^{l-i} a\left(x_{0}, \cdot\right)\right|_{s, A, i}^{(p)}<\infty,
$$

for $l \in N$ and $a=0$ for $\left|x_{0}\right|>T$.

As usual, for a symbol $a(x, \xi)$ we define a pseudodifferential operator $a(x, D)$,

$$
a(x, D) u(x)=\int e^{i x \xi} a(x, \xi) \hat{u}(\xi) \xi,
$$

where $\hat{u}(\xi)$ stands for a Fourier transform of $u(x)$ and $d \xi=(2 \pi)^{-(n+1)} d \xi$. We define $a_{A}(x, D)$ a transform of $a(x, D)$ by $e^{4}$ as follows

$$
a_{\Lambda}(x, D)=e^{\Lambda} a(x, D) e^{-\Lambda} .
$$

Lemma 2.3. Let $a\left(x^{\prime}, \xi^{\prime}\right)$ be in $\mathscr{A}_{s, A, h}^{(p)}$. Then $a_{A}\left(x^{\prime}, D^{\prime}\right)=e^{A} a\left(x^{\prime}, D^{\prime}\right) e^{-1}$ where $\Lambda=M\left(h+\left\langle D^{\prime}\right\rangle_{h}^{k}\right), \kappa=s^{-1}$ and $|M| \leq\left(24 n^{*} A^{*}\right)^{-1}$, is a pseudo-differential operator and its symbol $a_{A}\left(x^{\prime}, \xi^{\prime}\right)$ is in $S_{1,0, h}^{(p)}$ and satisfies

$$
\left|a_{1}\right|_{l}^{(p)} \leq C_{l}|a|_{s, A}^{(p)}, \quad\left[\frac{l}{1-\kappa}\right]+l+1,
$$

where $C_{l}$ is independent of $a$ and $h$ and $[\cdot]$ is a Gauss' symbol.

It's proof is refered to Proposition 2.3 in [4].

Definition 2.4. Denote by $\mathscr{A}^{(p, 1)}$ all functions $a\left(x^{\prime}, \xi^{\prime}\right)$ in $C^{\infty}\left(R^{n} \times R^{n}\right)$ such that

$$
\|a\|_{l}^{(p, h)}=\sup _{\substack{\xi \in R^{n} \\|\alpha+\beta| \leq l}} \frac{\left\|e^{\Lambda} a^{(\alpha)}(\beta)\left(\cdot, \xi^{\prime}\right)\right\|_{L^{2}\left(R_{x}^{n}\right)}^{n}}{\langle\xi\rangle_{h}^{p-|\alpha|}}<\infty
$$

for each $l \in N$, where $\Lambda=M\left(h+\left\langle D^{\prime}\right\rangle_{h}^{\kappa}\right), M \in R^{1}, 0<\kappa<1$ and $p \in R^{1} p \in R^{1}$.

Remark. If $a\left(x^{\prime}, \xi^{\prime}\right) \in \mathscr{A}^{(p, 1)}$ and $\Lambda=M\left(h+\left\langle D^{\prime}\right\rangle{ }_{h}^{*}\right), M \geq 0$, then it follows from Sobolev's lemma that $a\left(x^{\prime}, \xi^{\prime}\right) \in S_{1,0, h}^{(p)}$ and $|a|_{l}^{(p)} \leq C\|a\|_{l+n+1}^{(p)}$.

Proposition 2.5. Let $a\left(x^{\prime}, \xi^{\prime}\right)$ be in $\mathscr{A}^{(p, 1)}$, where $\Lambda=M\left(h+\left\langle D^{\prime}\right\rangle_{h}^{*}\right)$, $0<\kappa<1, M \geqslant 0$ and $p \in R^{1}$. Then $a_{1}\left(x^{\prime}, D^{\prime}\right)=e^{4} a\left(x^{\prime}, D^{\prime}\right) e^{-\Lambda}$ is a pseudo dif- 
ferential operators and it's symbol $a_{\Lambda}\left(x^{\prime}, \xi^{\prime}\right)$ belongs to $S_{1,0, h}^{(p)}$ and satisfies

$$
|a|_{l}^{(p)} \leq C_{l} \|\left. a\right|_{\left[\frac{i}{1-k}\right]+n+1+l} ^{(p, 4)}
$$

for each $l \in N$, where $C_{l}$ is independent of $h$ and [•] is a Gauss' symbol.

PROOF. It follows from [5] that the symbol $a_{A}(x, \xi)$ is given by

$$
a_{\Lambda}(x, \xi)=o s-\iint e_{R^{2 n}}^{-i y \eta+\Lambda(\xi+\eta)-\Lambda(\xi)} a(x+y, \xi) d y d \eta
$$

where $x, \xi \in R^{n}$ and $o s-\iint$ means an oscillatory integral. Hence we have by Taylor expansion,

$$
a_{\Lambda}(x, \xi)=\sum_{|\alpha|<N} a_{(\alpha)}(x, \xi) \lambda_{\alpha}(\xi)+r_{N}(x, \xi) \equiv r(x, \xi)+r_{N}(x, \xi)
$$

where $\lambda_{\alpha}(\xi)=\alpha !^{-1}\left(D_{\eta}^{\alpha} e^{\Lambda(\xi+\eta)-\Lambda(\xi)}\right)_{\eta=0}$ and

$$
r_{N}(x, \xi) \equiv \sum_{|\alpha|=N} \frac{1}{\alpha !} o s-\iint e^{-i y \eta+\Lambda(\xi+\eta)-\Lambda(\xi)} y^{\alpha} \int_{0}^{1} a_{(\alpha)}(x+\theta y, \xi) d \theta d y d \eta
$$

Noting that $\left|D_{\xi}^{\beta} \lambda_{\alpha}(\xi)\right| \leq C_{\alpha \beta}\langle\xi\rangle_{h}^{-(1-\kappa)|\alpha|-|\beta|}$,

$$
\begin{aligned}
\left|r_{(\beta)}^{(\alpha)}(x, \xi)\right| & \leq \sum_{|\gamma|<N} \sum_{\alpha^{\prime}+\alpha^{\prime \prime}=\alpha}\left(\begin{array}{c}
\alpha \\
\alpha^{\prime}
\end{array}\right)\left|a_{(\gamma+\beta)}^{\left(\alpha^{\prime}\right)}(x, \xi)\right|\left|\lambda_{r}^{\left(\alpha^{\prime \prime}\right)}(\xi)\right| \\
& \leq C_{\alpha}|a|_{|\alpha+\beta|+N+n}^{(p, A)}\langle\xi\rangle_{h}^{p-|\alpha|} .
\end{aligned}
$$

On the other hand, we have

$$
r_{N}(x, \xi)=\sum_{|\gamma|=N} \frac{1}{\gamma !} o s-\iint e^{-i y \eta+\Lambda(\xi+\eta)-\Lambda(\xi)} \mu_{r}(\xi+\eta) a_{(\gamma)}(x+\theta y, \xi) d \theta d y d \eta
$$

where

$$
\mu_{r}(\xi+\eta)=e^{-\Lambda(\xi+\eta)} D_{\eta}^{r} e^{\Lambda(\xi+\eta)}
$$

Hence

$$
\begin{aligned}
r_{N(\beta)}^{(\alpha)}(x, \xi) & =\sum_{|r|=N} \frac{1}{\gamma !} \sum_{\alpha^{\prime}+\alpha^{\prime \prime}=\alpha}\left(\begin{array}{c}
\alpha \\
\alpha^{\prime}
\end{array}\right) \iiint_{0}^{1} e^{-i y \eta} \mu_{\gamma}^{\left(\alpha^{\prime}\right)}(\xi+\eta) a_{(\gamma+\beta)}^{\left(\alpha^{\prime \prime}\right)}(x+\theta y, \xi) d \theta d y d \eta \\
& =\sum_{|\gamma|=N} \frac{1}{\gamma !} \sum_{\alpha^{\prime}+\alpha^{\prime \prime}=\alpha}\left(\begin{array}{c}
\alpha \\
\alpha^{\prime}
\end{array}\right) \int e^{\Lambda(\xi+\eta)-\Lambda(\xi)} \mu_{\gamma}^{\left(\alpha^{\prime}\right)}(\xi+\eta) F_{(\gamma+\beta)}^{\left(\alpha^{\prime \prime}\right)}(x, \xi, \eta,) d \eta,
\end{aligned}
$$

where

$$
\begin{gathered}
F_{(\gamma+\beta)}^{\left(\alpha^{\prime \prime}\right)}(x, \xi, \eta)=\int_{0}^{1} \int^{-i y \eta} a_{(r+\beta)}^{\left(\alpha^{\prime \prime}\right)}(x+\theta y, \xi) d y d \theta \\
=\int_{0}^{1} \int^{-i(y-x) \frac{\eta}{\theta}} a_{(\gamma+\beta)}^{\left(\alpha^{\prime \prime}\right)}(y, \xi) \frac{d y}{\theta^{n}} d \theta \\
=\int_{0}^{1} e^{i x\left(\frac{\eta}{\theta}\right) \theta^{-n}} \hat{a}_{(\gamma+\beta)}^{\left(\alpha^{\prime \prime}\right)}\left(\frac{\eta}{\theta}, \xi\right) d \theta
\end{gathered}
$$


here $\hat{a}(\eta, \xi)$ stands for a Fourier transform of $a(x, \xi)$ with respect to $x$. Noting that $\Lambda(\xi+\eta)-\Lambda(\xi)-\Lambda(\eta) \leq 0$ and

$$
\begin{aligned}
& \left|\mu_{\gamma}^{\left(\alpha^{\prime}\right)}(\xi+\eta)\right| \leq C\langle\xi+\eta\rangle_{h}^{-(1-x)\left(|\gamma|+\left|\alpha^{\prime}\right|\right)}, \\
& \left|r_{N(\beta)}^{(\alpha)}(x, \xi)\right| \leq C \sum \int\langle\xi+\eta\rangle^{-(1-\kappa) N} e^{\Lambda(\eta)}\left|F_{(\gamma+\beta)}^{\left(\alpha^{\prime \prime}\right)}(x, \xi, \eta)\right| d \eta \\
& \leqslant C\langle\xi\rangle_{h}^{-(1-x) N} \sum \int\langle\eta\rangle_{h}^{+}(1-x) N=e^{\Lambda(\eta)}\left|F_{(\gamma+\beta)}^{\left(\alpha^{\prime \prime}\right)}\right| d \eta \\
& \leqslant C\langle\xi\rangle_{h}^{-(1-\kappa) N} \iint_{0}^{1}\langle\eta\rangle_{h}^{+(1-\kappa) N} e^{\Lambda(\eta)} \theta^{-n}\left|\hat{a}_{(\gamma+\beta)}^{\left(\alpha^{\prime \prime}\right)}\left(\frac{\eta}{\theta}, \xi\right)\right| d \theta d \eta \\
& \leqslant C\langle\xi\rangle_{h}^{-(1-\kappa) N} \sum \int\langle\eta\rangle_{h}^{+(1-\kappa) N} e^{\Lambda(\eta)}\left|\hat{a}_{(\gamma+\beta)}^{\left(\alpha^{\prime \prime}\right)}(\eta, \xi)\right| d \eta \\
& \leqslant C\langle\xi\rangle_{h}^{-(1-\kappa) N+p}|a|_{\left[(1-\kappa)^{-1} N+(n+1) / 2\right]+1+N^{\prime}+|\alpha+\beta|}^{(p, A)},
\end{aligned}
$$

where we used $\langle\theta \eta\rangle_{h} \leqslant\langle\eta\rangle_{h}$ and $\Lambda(\theta \eta) \leqslant \Lambda(\eta)$ for $0 \leqslant \theta \leqslant 1$. If we take $N$ such that $(1-\kappa) N \geqslant|\alpha|$, we obtain

$$
\left|r_{N(\beta)}^{(\alpha)}(x, \xi)\right| \leqslant C\langle\xi\rangle_{h}^{p-|\alpha|}|| a \|_{[|\alpha|+(n+1) / 2]+N+|\alpha+\beta|+1}^{(p, 1)}
$$

which implies (2.2) with (2.3).

Next we indicate the boundedness of pseudodifferential operators in $W_{l, q, h}$ and $W_{l, q, h}^{(A)}$ which proof is refers to [5].

PRoposition 2.6. Let $a(x, \xi)$ be in $S_{\rho, \delta, h}^{(m, p)}$, where $0 \leqslant \delta<\rho \leqslant 1$ and $\delta<1 / 2$. Then there exists a positive number $C_{l, q}$ independent of $h$ such that

$$
\|a u\|_{W_{l, q, h}} \leqslant C_{l, q}|a|_{M_{0}(l, q)}^{(m, p)} \mid\|u\|_{W_{l+m, p+q, h}}
$$

for any $u$ in $W_{l+m, p+q, h}$, where

$$
\begin{aligned}
M_{0}(l, q) & =\left[(1-\delta / 2)^{-1}\left(|l|+|q|+l_{0}+n+2\right)\right]+l_{0} \\
l_{0} & =\left[\delta(n+2)(\rho-\delta)^{-1}\right]+1
\end{aligned}
$$

We denote by $C_{0}^{k}\left([-T, T]: \mathscr{A}^{(p, \Lambda)}\right)$, where $\Lambda=\left(T-x_{0}\right)\left(h+\left\langle D^{\prime}\right\rangle_{h}^{\kappa}\right)$ all functions $a\left(x, \xi^{\prime}\right)$ such that $x_{0} \in R^{1} \rightarrow a\left(x_{0}, x^{\prime}, \xi^{\prime}\right)$ in $\mathscr{A}^{(p, A)}$ is $k$ times continuously differentiable and $a\left(x, \xi^{\prime}\right)=0$ for $\left|x_{0}\right| \geq T$, and we put

$$
[a]_{k}^{(p, \Lambda)}=\sup _{\substack{0 \leqslant j \leqslant k \\\left|x_{0}\right| \leqslant T}}\left|\left(\partial / \partial x_{0}\right)^{j} a\left(x_{0}, \cdot, \cdot\right)\right|_{k-j}^{(p, 4)}
$$

Analogously we define $C_{0}^{k}\left([-T, T] ; \gamma_{A}^{(s)}\left(R^{n}\right)\right)$, all functions $a(x)$ such that $x_{0} \rightarrow a\left(x_{0}, x^{\prime}\right)$ in $\gamma_{A}^{(s)}\left(R^{n}\right)$ is $k$ times continuously differentiable and $a=0$ for $\left|x_{0}\right| \geq T$, and denote 


$$
[a]_{s, A, k}=\sup _{\substack{0 \leqslant j \leqslant k \\\left|x_{0}\right| \leqslant T}}\left|\left(\partial / \partial x_{0}\right)^{j} a\left(x_{0}, \cdot\right)\right|_{s, A, k-j} .
$$

Then Proposition 2.5 and 2.6 imply directly

Proposition 2.7. Let $a\left(x, \xi^{\prime}\right)$ be in $C_{0}^{k}\left([-T, T] ; \mathscr{A}^{(p, 1)}\right.$, where $\Lambda=$ $\left(T-x_{0}\right)\left(h+\left\langle D^{\prime}\right\rangle_{h}^{k}\right)$. Then we have $C_{l, q}$ independent of $h$ such that

$$
\|a u\|_{W_{l, q, h}^{(A)}} \leqslant C_{l, q}[a]_{M_{i}^{\prime}(l, q)}^{(p, h)}\|u\|_{W_{l, q+p, h}^{(\lambda)}}
$$

for $u$ in $W_{l, q+p, h}^{(1)}$ and $l, q \in R^{1}$ such that $M_{\Lambda}(l, q) \leqslant k$, where

$$
M_{A}(l, q)=\left[M_{0}(l, q)(1-\kappa)^{-1}\right]+1+n+M_{0}(l, q),
$$

and $M_{0}(l, q)$ is given by (2.4).

Proposition 2.8. Let $a(x)$ be in $C_{0}^{k}\left([-T, T] ; \gamma_{A_{0}}^{(s)}\left(R^{n}\right)\right)$. Then if $0 \leq T \leqslant\left(24 n^{*} A_{0}^{x}\right)^{-1}$ and $\kappa=s^{-1}$, we have

$$
\|a u\|_{W_{l, q, h}^{(A)}} \leqslant C[a]_{s, A_{0}, M_{\Lambda}(l, q)}\|u\|_{w_{l, q, h}^{(\Lambda)}},
$$

for any $u$ in $W_{l, q, h}^{(\Lambda)}, M_{\Lambda}(l, q) \leqslant k$, where $M_{\Lambda}(l, q)$ given by (2.5) and $\Lambda=$ $\left(T-x_{0}\right)\left(h+\left\langle D^{\prime}\right\rangle_{h}^{c}\right)$.

Applying the above Propositions, we calculate a norm of composite functions in $W_{l, q, h}^{(1)}$.

Proposition 2.9. Let $a(x, y)$ be in $C^{k}\left(R^{n+1} \times B_{R_{0}}\right)$, where $B_{R_{0}}=\left\{y \in R^{r}\right.$; $\left.|y| \leqslant R_{0}\right\}$ and satisfies

$$
\begin{aligned}
& a(x, 0)=0 \\
& a(x, y)=a_{0}(y), \quad\left|x_{0}\right|>T, \\
& \quad\left|D_{x}^{\alpha} D_{y}^{\beta} a(x, y)\right| \leqslant C_{\alpha_{0}} A_{\alpha_{0}}^{\left|\alpha^{\prime}\right|+|\beta|}\left|\alpha^{\prime}\right| !|s| \beta \mid !,
\end{aligned}
$$

for any $\alpha=\left(\alpha_{0}, \alpha^{\prime}\right) \in N^{n+1}, \alpha_{0} \leq k, \beta \in N^{r}$ and $(x, y) \in R^{n+1} \times B_{R_{0}}$. Assume that $0 \leqslant T \leqslant\left(24 n^{x} A_{0}^{x}\right)^{-1}$ and $v=\left(v_{1}, \cdots, v_{r}\right)$ is in $\left(W_{l, q, h}^{(\Lambda)}\right)^{r}$, where $\Lambda=\left(T-x_{0}\right)(h+$ $\left.\left.\left\langle D^{\prime}\right\rangle\right\rangle_{h}^{\kappa}\right), \kappa=s^{-1}$ and $q \in R^{1}, M_{\Lambda}(l, q) \leq k$ and satisfies

$$
\|\boldsymbol{v}\|_{W_{l, q, h}^{(\lambda)}}=\left\{\sum_{i=1}^{r}\left\|\boldsymbol{v}_{i}\right\|_{W_{l, q, h}^{(1)}}^{2}\right\}^{1 / 2} \leqslant\left(2 r A_{0}\right)^{-1},
$$

then $a \circ v(x)=a(x, v(x))$ is in $W_{l, q, h}^{(1)}$ and satisfies

$$
\|a \circ v\|_{W_{l, q, h}^{(\lambda)}} \leqslant C\left(A_{0}\right),
$$

where $C\left(A_{0}\right)$ is independent of $h$.

Proof. By Taylor's expansion, we have 


$$
a(x, y)=\sum_{\beta \neq 0} a_{(\beta)}(x) y^{\beta} / \beta !
$$

where $a_{(\beta)}(x)=(\partial / \partial y)^{\beta} a(x, 0)$. Then it follows from $(2.6)$ : that $a_{(\beta)}(x)$ is in $C_{0}^{\infty}\left([-T, T] ; \gamma_{A_{0}}^{(s)}\right.$ and satisfies

$$
\left[a_{(\beta)}\right]_{s, A_{0}, l}<C_{l} A_{0}^{|\beta|}|\beta| ! \text {. }
$$

is

Hence we have by Lemma 1. 1, Proposition 2.7 and (2:7)

$$
\begin{aligned}
\|a \circ v\|_{W_{l, q, h}^{(A)}} & \leqslant C_{l} \sum_{\beta \neq 0} \frac{1}{\beta !}\left[a_{(\beta)}\right]_{s, A_{0}, M_{\Lambda}(l, q)}\left(\|v\|_{W_{l, q, h}^{(A)}}\right)^{|\beta|} \\
& \leqslant C_{l} \sum_{j=1}^{\infty}\left(r A_{0}\|v\|_{W_{l, q, h}^{(A)}}\right)^{j} \leqslant C_{l}
\end{aligned}
$$

where $C_{l}$ is independent of $h$.

Let $Q$ be a cube consisting of $x$ in $R^{n+1}$ with each $\left|x_{j}\right|<1 j=1, \cdots, n+1$, and $Q_{k}(k=1, \cdots)$ an enumeration of tranlates of $Q$ centered at the integer lattice point $g^{(k)}$ of $R^{n+1}$. Let $\theta(x)$ in $C_{0}^{\infty}\left(R^{n+1}\right)$ be supported in $Q$, and $\varphi_{j}(x)=$ $\theta\left(x-g^{(j)}\right) \times\left[\sum_{k=1}^{\infty} \theta\left(x-g^{(k)}\right)^{2}\right]^{-1 / 2}$ and $\left.\chi(x) \in C_{0}^{\infty} R^{n+1}\right)$ such that $\chi=1$ on $\mathscr{B}_{R_{1}}=$ $\left\{x \in R^{1},|x| \leq R_{1}\right\}$. We define $\varphi_{j k}\left(x, \xi^{\prime}\right), j, k=0,1, \cdots$,

$$
\begin{aligned}
\varphi_{00}\left(x, \xi^{\prime}\right) & =(1-\chi(x))\left(\chi^{2}+(1-\chi)^{2}\right)^{-1 / 2} \\
\varphi_{0 j}=\varphi_{k 0} & =0, \quad j, k=1,2, \cdots \\
\varphi_{j k}\left(x, \xi^{\prime}\right) & =\varphi_{j}\left(\left(x \Lambda-g^{(k)}\right)\left\langle\xi^{\prime}\right\rangle_{h}^{o}\right) \varphi_{k}(x) \chi(x)\left(\chi^{2}+(1-\chi)^{2}\right)^{-1 / 2} \\
j, k & =1,2, \cdots,
\end{aligned}
$$

where $x_{\Lambda}=\left(x_{0}, x^{\prime}+\operatorname{grad}_{\xi^{\prime}}\left\langle\xi^{\prime}\right\rangle_{h}^{\kappa}\right)$ (if there is no confusion we write also $x_{A}=$ $\left.e^{A} x e^{-\Lambda}\right)$ and $0<\sigma<1 / 2$. Then we have

LEMMA 2. 10. $\left\{\varphi_{j k}\left(x, \xi^{\prime}\right)\right\}_{j, k=0,1, \ldots}$ satisfies

$$
\sum_{k=0}^{\infty} \sum_{j=0}^{\infty} \varphi_{j k}\left(x, \xi^{\prime}\right)^{2}=1, \quad \text { on } R^{n+1} \times R^{n}
$$

on $R^{n+1} \times R^{n}$, for $(\alpha, \beta) \in N^{n+1} \times N^{n}$.

It follows from $(2.8)$ that $\varphi_{j k(\beta)}^{(\alpha)}\left(x, \xi^{\prime}\right)$ is in $S_{1-\sigma, \sigma, h}^{(0, \sigma|\beta|-(1-\sigma)|\alpha|)}$ uniformly in $(j, k)$, if we take $0<\sigma<1 / 2$. Therefore we obtain the following proposition by Lemma 2.1 and Proposition 2.6.

Proposition 2. 11. Let $\left\{\varphi_{j k}(x, D)\right\}$ be pseudo-differential operators with symbols $\left\{\varphi_{j k}\left(x, \xi^{\prime}\right)\right\}$. and $0<\sigma<1 / 2$. Then there are positive numbers $C$ and $h_{0}$ such that 


$$
C^{-1}\|u\|_{W_{l, q, h}}^{2} \leqslant \sum_{j, k}\left\|\varphi_{j k} u\right\|_{W_{l, q}}^{2} \leqslant C\|u\|_{W_{l, q}, h}^{2}
$$

for any $u$ in $W_{l, q, h}$ and $h \geqslant h_{0}$.

We put

$$
x_{j k}=x_{j k}\left(D^{\prime}\right)=g^{(k)}+g^{(j)}\left\langle D^{\prime}\right\rangle_{h}^{-\sigma}, \quad j, k=1,2, \cdots .
$$

If there is no confusion, we write also $x_{j k}=g^{(k)}+g^{(j)}\left\langle\xi^{\prime}\right\rangle_{h}^{-a}$. Since the support of $\varphi_{j k}\left(x, \xi^{\prime}\right)$ is contained in $\left\{\left(x, \xi^{\prime}\right) \in R^{n+1} \times R^{n} ;\left|x_{A}-x_{j k}\right| \leqslant\left\langle\xi^{\prime}\right\rangle_{h}^{-\sigma}\right\}, \quad\left(x_{A}-x_{j k}\right)^{r}$ $\varphi_{j k}\left(x, \xi^{\prime}\right)$ is in $S_{1+i \sigma, \sigma, h}^{(0,-|r| \sigma)}$ uniformly in $(j, k)$. Hence we have

Proposition 2.12. (c.f. [5]). Assume that

$$
a_{j k}(x, \xi)=\sum_{|r|=d}\left(x_{\Lambda}-x_{j k}\right)^{r} b_{j k_{\gamma}}(x, \xi)
$$

where $b_{j k}(x, \xi)$ is in $S_{1,0, h}^{(m, p)}$ uniformly in $(j, k)$. Then we have a positive number $C_{l, q}$ independent of $h$ such that

$$
\begin{aligned}
& \sum_{j=1}^{\infty} \sum_{k=1}^{\infty}\left\|a_{j k} \varphi_{j k} u\right\|_{W_{l, q, h}}^{2} \leqslant C\left[\sup _{j, k}\left|b_{j k}\right|_{M_{1}(l, q)}^{(m, p)}\right]^{2}\|u\|_{W_{l+m, p+q-d \sigma, h}}^{2}, \\
& \sum_{j=1}^{\infty} \sum_{k=1}^{\infty}\left\|\left[a_{j k}, \varphi_{j k}\right] u\right\|_{W_{l}, q, h}^{2} \leqslant C\left[\sup _{j, k}\left|b_{j k}\right|_{M_{1}(l, q)}^{(m, p)}\right]^{2}\|u\|_{W_{l+m, p+q-d_{\sigma+\sigma-1, h}}^{2},}
\end{aligned}
$$

where

$$
M_{1}(l, q)=M_{0}(l, q)+2\left[n 2^{-1}+1\right]+2\left[\frac{|m|+|q|+n+M_{0}(l, q)}{2(1-\sigma)}+1\right]
$$

and $M_{0}(l, q)$ is given by (2.4).

Lemma 2. 13. Let $a\left(x, \xi^{\prime}\right)$ be in $C_{0}^{\infty}\left([-T, T] ; \mathscr{A}^{(p, 1)}\right)$ or in $C_{0}^{\infty}([-T, T]$; $\mathscr{A}_{\left.s, A_{0}, h\right)}^{(p)}$, where $\Lambda=\left(T-x_{0}\right)\left(h+\left\langle D^{\prime}\right\rangle_{h}^{k}\right)$. Then

$$
\begin{aligned}
& a\left(x, D^{\prime}\right)-a\left(x_{j k}, D^{\prime}\right)=\sum_{|\alpha|=1} a_{(\alpha)}\left(x_{j k}, D^{\prime}\right)\left(x-x_{j k}\right)^{\alpha} \\
& \quad+\sum_{|\alpha|=2} Q_{j k \alpha}(a)\left(x, D^{\prime}\right)\left(x-x_{j k}\right)^{\alpha}+R_{j k}(a)\left(x, D^{\prime}\right),
\end{aligned}
$$

where $x_{j k}=x_{j k}\left(D^{\prime}\right)$ is given by (2.9) and $Q_{j k \alpha}(a)\left(x, \xi^{\prime}\right)$ in $C_{0}^{\infty}([-T, T]$; $\left.\mathscr{A}^{(p, 1)}\right)\left(\right.$ or $\left.C_{0}^{\infty}\left([-T, T] ; \mathscr{A}_{s, A_{0}, h}^{(p)}\right)\right)$ and $R_{j k}(a)\left(x, \xi^{\prime}\right)$ in $C_{0}^{\infty}\left([-T, T] ; \mathscr{A}^{(p-1,1)}\right)$ (or $C_{0}^{\infty}\left([-T, T] ; \mathscr{A}_{s, A_{0}, h}^{(p-1)}\right)$ uniformly in $(j, k)$ respectively, and it holds for $l \in N$,

$$
\begin{array}{ll}
\sup _{j, k, \alpha}\left[Q_{j k \alpha}(a)\right]_{i}^{(p, 1)} \leqslant C_{l}[a]_{l+2}^{(p, \Lambda)} & \left(\text { or }[a]_{a, A_{0}, l+2}^{(p)}\right) \\
\sup _{j, k}\left[R_{j k}(a)\right]_{l}^{(p-1,1)} \leqslant C_{l}[a]_{l+2}^{(p, \Lambda)} & \left(\text { or }[a]_{s, A_{0}, l+2}^{(p)}\right)
\end{array}
$$

where $C_{l}$ is independent of $h$. 
Proof. By Taylor expansion, we have

$$
\begin{aligned}
& a\left(x, \xi^{\prime}\right)-a\left(x_{j k}\left(\xi^{\prime}\right), \xi^{\prime}\right)=\sum_{|\alpha|=1} a_{(\alpha)}\left(x_{j k}, \xi^{\prime}\right)\left(x-x_{j k}\right)^{\alpha} \\
& \quad+\sum_{|\alpha|=2} \frac{1}{\alpha !} \int_{0}^{1} a_{(\alpha)}\left(x_{j k}+\theta\left(x-x_{j k}\right), \xi^{\prime}\right)\left(x-x_{j k}\right)^{\alpha} d \xi .
\end{aligned}
$$

Hence we obtain

$$
Q_{i k \alpha}(a)\left(x, \xi^{\prime}\right)=\int_{0}^{1} a_{(\alpha)}\left(x_{j k}+\theta\left(x-x_{j k}\right), \xi^{\prime}\right) d \theta / \alpha !
$$

and

$$
\begin{aligned}
& R_{j k}(a)\left(x, \xi^{\prime}\right)=\sum_{|\alpha|=1} \partial_{\xi}^{\alpha}\left(a_{(\alpha)}\left(x_{j k}(\xi), \xi^{\prime}\right)\right) \\
& \quad+\sum_{|\alpha|=2} o s-\iint e^{-i y \eta^{\prime}} Q_{j k \alpha}(a)\left(x, \xi^{\prime}+\eta^{\prime}\right)\left(x+y-x_{j k}\right) d y d \eta^{\prime} \\
& \quad-\sum_{|\alpha|=2} Q_{j k_{\alpha}}(a)\left(x, \xi^{\prime}\right)\left(x-x_{j k}\right)^{\alpha},
\end{aligned}
$$

which satisfy (2.12) and (2.13) respectively.

Noting that $e^{4} a\left(x_{j k}\left(D^{\prime}\right), D^{\prime}\right) e^{-4}=a\left(x_{j k}, D^{\prime}\right)$, we have,

Corollary 2.14. Let $a\left(x, \xi^{\prime}\right)$ be in $C_{0}^{\infty}\left([-T, T] ; \mathscr{A}^{(p, t)}\right)$ or $C_{0}^{\infty}([-T, T]$; $\left.\mathscr{A}_{s, A_{0}, h}^{(p)}\right)$, where $\Lambda=\left(T-x_{0}\right)\left(h+\left\langle D^{\prime}\right\rangle_{h}^{k}\right)$. Then we have

$$
\begin{gathered}
a_{\Lambda}\left(x, D^{\prime}\right)=a\left(x_{j k}\left(D^{\prime}\right), D^{\prime}\right)+\sum_{|\alpha|=1} a_{(\alpha)}\left(x_{j k}, D^{\prime}\right)\left(x_{\Lambda}-x_{j k}\right)^{\alpha} \\
+\sum_{|\alpha|=2} Q_{j k \alpha}(a)_{\Lambda}\left(x, D^{\prime}\right)\left(x_{\Lambda}-x_{j k}\right)^{\alpha}+S_{j k}(a)_{\Lambda}\left(x, D^{\prime}\right),
\end{gathered}
$$

where $Q_{j k \alpha}(a)_{\Lambda}\left(x, \xi^{\prime}\right)$ is in $S_{1,0, h}^{(0, p)}$ and $S_{j k}(a)_{\Lambda}\left(x, \xi^{\prime}\right)$ in $S_{1,0, h}^{(0, p-1)}$ uniformly in $(j, k)$ and satisfy

$$
\begin{aligned}
& \left|Q_{j k \alpha}(a)_{\Lambda}\right|_{l}^{(0, p)} \leqslant C_{l}[a]_{\left[(l+2)(1-\varepsilon)-{ }^{1}\right]+n+l+2}^{(0, p)}, \\
& \quad\left(\text { or }[a]_{\left.s, A_{0},[(l+2)(1-\kappa))^{-1}\right]+n+l+2}^{(p)}\right) \\
& \left|S_{j k}(a)_{\Lambda}\right|_{l}^{(0, p-1)} \leqslant C_{l}[a]_{\left.[(l+2)(1-\kappa))^{-1}\right]+n+l+2}^{(p, A)} \\
& \quad\left(\text { or }[a]_{\left.s, A_{0},[(l+2)(1-\kappa))^{-1}\right]+n+l+2}^{(p)} .\right.
\end{aligned}
$$

Proof. (2.15) and (2.16) follow from (2.12), (2.13) and Proposition 2.5.

Lemma 2.15. Let $a\left(x, \xi^{\prime}\right)$ be in $C_{0}^{\infty}\left([-T, T] ; \mathscr{A}^{(p, A)}\right)$ or $C_{0}^{\infty}([-T, T]$; $\mathscr{A}_{s, A_{0}, h}^{(p)}$, where $\Lambda=\left(T-x_{0}\right)\left(h+\left\langle D^{\prime}\right\rangle_{h}^{\kappa}\right)$. Then we have,

$$
\begin{aligned}
{\left[a_{1}, \varphi_{j k}\right] } & =\sum_{|\alpha|=1}\left\{a_{(\alpha)}\left(x_{j k}, D^{\prime}\right)+\sum_{|\beta|=1} a_{(\beta)}\left(x_{j k}, D^{\prime}\right)\left(x_{j k}^{\beta}\right)^{(\alpha)}\right\} \varphi_{j k(\alpha)}\left(x, D^{\prime}\right) \\
& +T_{j k}(a)\left(x, D^{\prime}\right),
\end{aligned}
$$


where $T_{j k}(a)\left(x, \xi^{\prime}\right)$ is in $S_{1-\sigma, \sigma, h}^{(0, p-1)}$ and satisfies

$$
\begin{gathered}
\sum_{j, k}\left\|T_{j k}(a) u\right\|_{W_{l, q, h}}^{2} \leqslant C_{l, q}\left\{[a]_{M_{2}(l, q)}^{(p, A)}\|u\|_{W_{l, q+p-1, h}}\right\}^{2} \\
\quad\left(\text { or }\left\{[a]_{s, A_{0}, M_{1}(l, q)}^{(p)}\|u\|_{W_{l, q+p-1, h}}\right\}^{2}\right)
\end{gathered}
$$

where

$$
M_{2}(l, q)=\left[\left(M_{1}(l, q)+2\right)(1-\kappa)^{-1}\right]+n+M_{1}(l, q)+2,
$$

and $M_{1}(l, q)$ is given by $(2.11)^{\prime}$.

Proof. We put

$$
\begin{aligned}
c_{j k}\left(x, D^{\prime}\right) & =a_{\Lambda}\left(x, D^{\prime}\right)-a\left(x_{j k}, D^{\prime}\right) \\
& =\sum_{|\alpha|=1} a_{(\alpha)}\left(x_{j k}, D^{\prime}\right)\left(x_{\Lambda}-x_{j k}\right)^{\alpha} \\
& +\sum_{|\alpha|=2} Q_{j k_{\alpha}}(a)_{\Lambda}\left(x, D^{\prime}\right)\left(x_{\Lambda}-x_{j k}\right)^{\alpha}+S_{j k}(a)_{\Lambda}\left(x, D^{\prime}\right) .
\end{aligned}
$$

Then it follows from (2.15), (2.16) and (2.11) that

$$
\begin{aligned}
& \sum_{j, k}\left\|\left[C_{j k}, \varphi_{j k}\right] u\right\|_{W_{l}, q, h}^{2} \leqslant C\left\{\sup _{j, k,|\alpha|=1}\left|a_{(\alpha)}\left(x_{j k}, \cdot\right)\right|_{M_{1}(l, q)}^{(0, p-1)}\right\}^{2}\|u\|_{W_{l, q+p-1 . h}}^{2} \\
& +C_{l, q}\left\{\sup _{j, k,|\alpha|=2,}\left|Q_{j k \alpha}(a)_{1}\right|_{M_{1}(l, q)}^{(0, p)}\|u\|_{W_{l, q+p-\sigma-1, h}}\right\}^{2} \\
& +C_{l, q}\left\{\sup _{j, k}\left|S_{j k}(a)_{\Lambda}\right|\left\|_{M_{1}(l, q)}^{(0, p)}|| u\right\|_{W_{l, q+p-2+\sigma, h}}\right\}^{2} \\
& \leqslant C_{l, q}\left\{[a]_{M_{2}(l, q)}^{(p, h)}\|u\|_{W_{l, q+p-1, h}}=2 .\right.
\end{aligned}
$$

On the other hand,

$$
\left(\left[a\left(x_{j k}\left(D^{\prime}\right), D^{\prime}\right), \varphi_{j k}\right]\right)\left(x, \xi^{\prime}\right)=\sum_{|\alpha|=1} a_{j k}^{(\alpha)}\left(\xi^{\prime}\right) \varphi_{j k(\alpha)}\left(x, \xi^{\prime}\right)+\tilde{c}_{j k}\left(x, \xi^{\prime}\right),
$$

where $a_{j k}\left(\xi^{\prime}\right)=a\left(x_{j k}\left(\xi^{\prime}\right), \xi^{\prime}\right)$ and

$$
\begin{aligned}
& \tilde{c}_{j k}\left(x, \xi^{\prime}\right)=o s-\iint e^{-i y^{\prime} \eta^{\prime}} a_{j k}\left(\xi^{\prime}+\eta^{\prime}\right) \varphi_{j k}\left(x+y, \xi^{\prime}\right) d y^{\prime} d \eta^{\prime} \\
& \quad-\sum_{|\alpha|=1} a_{j k}^{(\alpha)}\left(\xi^{\prime}\right) \varphi_{j k}\left(x, \xi^{\prime}\right) \\
& =o s-\iint e^{-i y^{\prime} \eta^{\prime}} \sum_{|\alpha|=2} a_{j k}^{(\alpha)}\left(\xi^{\prime}+\eta^{\prime}\right) \int_{0}^{1} \varphi_{j k}\left(x+\theta y, \xi^{\prime}\right) d \theta d y^{\prime} d \eta^{\prime} .
\end{aligned}
$$

Noting that $a_{j k}\left(\xi^{\prime}\right)$ is in $S_{1,0, h)}^{(0, p)}$ uniformly in $(j, k)$, and $\varphi_{j k}\left(x, \xi^{\prime}\right)$ satisfies (2.8), we obtain by virtue of Proposition 2.6,

$$
\begin{aligned}
\sum_{j, k}\left\|\tilde{c}_{j k} u\right\|_{W_{l, q, h}}^{2} & \leqslant C_{l, q}\left\{\sup \left|a_{j k}\right|_{\left.M_{1} l, q, q\right)}^{(0, p)}\|u\|_{W_{l, q+p-2(1-o), h}}\right\}^{2} \\
& \leqslant C_{l, q}\left\{[a]_{\mathcal{M}_{2}(l, q)}^{(p,,)}\|u\|_{W_{l, q+p-2(1-\sigma), h}}\right\}^{2},
\end{aligned}
$$


which implies (2.18) with (2.20).

Thus summarizing up, we obtain,

Proposition 2.16. Let $a(x, \xi)=\sum_{i=0}^{m} a_{i}\left(x, \xi^{\prime}\right) \xi_{0}^{m-i}$ be polynomial in $\xi_{0}$ of which coefficients $a_{i}\left(x, \xi^{\prime}\right)=b_{i}\left(x, \xi^{\prime}\right)+c_{i}\left(x, \xi^{\prime}\right)+d_{i}\left(\xi^{\prime}\right)$, where $b_{i}$ is in $C_{0}^{\infty}([-T, T]$; $\left.\mathscr{A}^{(i, 1)}\right), c_{i}$ in $C_{0}^{\infty}\left([-T, T] ; \mathscr{A}_{s, A_{0}, h}^{(i)}\right)$ and $d_{i}$ in $\mathrm{S}_{1,0, h}^{(i)}$ respectively, and $\Lambda=$ $\left(T-x_{0}\right)\left(h+\left\langle D^{\prime}\right\rangle_{h}^{k}\right), \kappa=s^{-1}$ and $0 \leqslant T \leqslant\left(24 n^{\kappa} A_{0}^{k}\right)^{-1}$. Then $a_{1}(x, \xi)$ is in $S_{1,0, h}^{(m, 0)}$ and satisfies,

$$
\begin{aligned}
& \left\{a\left(x_{j k}\left(D^{\prime}\right), D_{A}\right)-a_{\Lambda}(x, D)\right\} \varphi_{j k}\left(x, D^{\prime}\right)+\left[a_{A}, \varphi_{j k}\right] \\
& \quad=\sum_{|\alpha|=1} a_{(\alpha)}\left(x_{j k}, D_{\Lambda}\right)\left(x_{A}-x_{j k}\right)^{\alpha} \varphi_{j k}\left(x, D^{\prime}\right) \\
& \quad+\sum_{|\alpha|=1}\left\{a^{(\alpha)}\left(x_{j k}, D_{\Lambda}\right)+\sum_{|\beta|=1} a_{(\beta)}\left(x_{j k}, D_{A}\right) x_{j k}^{\beta(\alpha)}(D)\right\} \varphi_{j k(\alpha)}(x, D) \\
& \quad+R_{j k}(a)(x, D),
\end{aligned}
$$

where $R_{j k}(a)(x, \xi)$ is in $S_{1-\sigma, \sigma, h}^{(m,-1)}$ and

$$
\begin{gathered}
\sum_{j, k}\left\|R_{j k}(a) u\right\|_{W_{l, q, h}}^{2} \leqslant C_{l, q}\left\{\sup _{i}\left[b_{i}\right]_{M_{2}(l, q)}^{(i, q)}+\sup _{i}\left[c_{i}\right]_{M_{2}(l, q)}^{(i)}\right. \\
\left.+\sup _{i}\left|d_{i}\right|_{M_{2}(l, q)}^{(i)}\right\}^{2}\|u\|_{W_{l+m, q-1, h}}^{2}
\end{gathered}
$$

where $M_{2}(l, q)$ is given by (2.19).

Proof. We have

$$
\begin{aligned}
a_{\Lambda}(x, D) & =\sum_{i=0}^{m} e^{\Lambda} a_{i}\left(x, D^{\prime}\right) D_{0}^{m-i} e^{-\Lambda} \\
& =\sum_{i=0}^{m} a_{i \Lambda}\left(x, D^{\prime}\right)\left(D_{0 \Lambda}\right)^{m-i}
\end{aligned}
$$

Since $a_{i \Lambda}\left(x, \xi^{\prime}\right)$ is in $S_{1,0, h}^{(0, i)}$ and $\xi_{0 \Lambda}^{m-i}=\left(\xi_{0}-\sqrt{-1}\left\langle\xi^{\prime}\right\rangle_{h}^{k}\right)^{m-i}$ in $S_{1,0, h}^{(m-i, 0)}$, it is clearly that $a_{A}(x, \xi)$ is in $S_{1,0, h}^{(m, 0)}$. Moreover for $a_{i \Lambda}\left(x, D^{\prime}\right),(2.14)$ and (2.17) are valid. Noting that $e^{4} a\left(x_{j k}\left(D^{\prime}\right), D\right) e^{-\Lambda}=a\left(x_{j k}, D_{1}\right)$, we have

$$
\begin{aligned}
a_{\Lambda}(x, & D)-a\left(x_{j k}, D_{\Lambda}\right)=\sum_{i=0}^{m}\left(b_{i \Lambda}\left(x, D^{\prime}\right)-b_{i}\left(x_{j k}, D^{\prime}\right)\right) D_{0 \Lambda}^{m-i} \\
& +\sum_{i=1}^{m}\left(c_{i \Lambda}\left(x, D^{\prime}\right)-c_{i}\left(x_{j k}, D^{\prime}\right)\right) D_{0 \Lambda}^{m-i} \\
& =\sum_{|\alpha|=1} a_{(\alpha)}\left(x_{j k}, D_{\Lambda}\right)\left(x_{\Lambda}-x_{j k}\right)^{\alpha} \\
& +\sum_{i=0}^{m}\left\{\sum_{|\alpha|=2} Q_{j k \alpha}\left(b_{i}+c_{i}\right)_{\Lambda}\left(x, D^{\prime}\right)\left(x_{\Lambda}-x_{j k}\right)^{\alpha}+S_{j k}\left(b_{i}+c_{i}\right)_{\Lambda}\right\}\left(D_{0 \Lambda}\right)^{m-i} \\
& =\sum_{|\alpha|=1} a_{(\alpha)}\left(x_{j k}, D_{\Lambda}\right)\left(x_{\Lambda}-x_{j k}\right)^{\alpha}+\tilde{S}_{j k}(x, D)
\end{aligned}
$$


It follows from Corollary 2.14 that $\widetilde{S}_{j k}(x, D) \varphi_{j k}\left(x, D^{\prime}\right)$ satisfies $(2.22)$. On the other hand we have

$$
\left[a_{1}, \varphi_{j k}\right]=\left[a_{\Lambda}-a\left(x_{j k}, D_{\Lambda}\right), \varphi_{j k}\right]+\left[a\left(x_{j k}, D_{\Lambda}\right), \varphi_{j k}\right] .
$$

It follows from that Proposition 2.12 that $\left[a_{A}-a\left(x_{j k}, D_{A}\right), \varphi_{j k}\right]$ satisfies (2.22). The last term is

$$
\begin{aligned}
& {\left[a\left(x_{j k}, D_{\Lambda}\right), \varphi_{j k}\right]=\sum_{i=0}^{m}\left\{a_{i}\left(x_{j k}, D^{\prime}\right)\left[D_{04}^{m-i}, \varphi_{j k}\right]\right.} \\
& \left.\quad+\left[a_{i}\left(x_{j k}, D^{\prime}\right), \varphi_{j k}\right] D_{04}^{m-i}\right\} .
\end{aligned}
$$

Noting that $\left[D_{0 \Lambda}^{m-i}, \varphi_{j k}\right]=(m-i)\left(D_{01}\right)^{m-i}\left(D_{0} \varphi_{j k}\right)\left(x, D^{\prime}\right)+\varphi_{j k i}\left(x, D^{\prime}\right)$, where $\varphi_{j k i}(x, \xi)$ is in $S_{1-\sigma, \sigma, h}^{(m-i-2,2 \sigma)}$ uniformly in $(j, k)$ and satisfies from $(2.8)$,

$$
\sum_{j, k}\left|\varphi_{j k i(\beta)}^{(\alpha)}\left(x, \xi^{\prime}\right)\right| \leqslant C_{\alpha \beta}\langle\xi\rangle_{h}^{m-i-2-\alpha_{0}}\left\langle\xi^{\prime}\right\rangle_{h}^{2 \sigma-(1-o)\left|\alpha^{\prime}\right|+\sigma|\beta|} .
$$

Hence we have from (2.24) and (2.17),

$$
\begin{aligned}
{\left[a\left(x_{j k}, D_{A}\right), \varphi_{j k}\right] } & =\sum_{|\alpha|=1}\left\{a^{(\alpha)}\left(x_{j k}, D_{A}\right)+\sum_{|\beta|=1} a_{(\beta)}\left(x_{j k}, D_{A}\right) x_{j k}^{\beta(\alpha)}\right\} \\
& \times \varphi_{j k(\alpha)}\left(x, D^{\prime}\right)+\widetilde{T}_{j k}(a)(x, D),
\end{aligned}
$$

where

$$
\begin{gathered}
\tilde{T}_{j k}(a)(x, D)=\sum_{i=0}^{m}\left\{a_{i}\left(x_{j k}, D^{\prime}\right) \varphi_{j k i}(x, D)+T_{j k}\left(a_{i}\right)\right\} D_{0 .}^{m-i} \\
+\sum_{i=0}^{m} \sum_{|\beta|=1} a_{(\beta)}\left(x_{j k}, D_{A}\right)\left[x_{j k}^{\beta(\alpha)} \varphi_{j k(\alpha)}, D_{0.1}^{m-i}\right] .
\end{gathered}
$$

Hence

$$
\begin{aligned}
& \sum_{j, k}\left\|\tilde{T}_{j k}(a) u\right\|_{W_{l, q, h}}^{2} \leqslant C_{l, q} \sum_{j, k} \sum_{i=0}^{m}\left\{\left(\left|a_{i}\right|_{0}^{(i)}\left\|\varphi_{j k i} u\right\|_{W_{l+i, q, h}}\right)^{2}\right. \\
& \quad+\sum_{|\beta|=1}\left(\left|a_{i}\right|_{1}^{(i)}\left\|\left[x_{j k}^{\beta(\alpha)} \varphi_{j k(\alpha)}, D_{0.1}^{m-i}\right] u\right\|_{W_{l+i, q, h}}\right)^{2} \\
& \left.\quad+\left\|T_{j k}\left(a_{i}\right) D_{01}^{m-i} u\right\|_{W_{l, q, h}}^{2}\right\} .
\end{aligned}
$$

Since $x_{j k}^{\beta(\alpha)}\left(\xi^{\prime}\right)$ is in $S_{1,0, h}^{(0,-|\alpha|)}$ uniformly in $(j, k)$, it follows from Proposition 2.11 that

$$
\sum_{j, k}\left\|\left[x_{j k}^{\beta(\alpha)} \varphi_{j k(\alpha)}, D_{0,}^{m-i}\right] u\right\|_{W_{l+i, q, h}}^{2} \leqslant C_{l, q}\|u\|_{W_{l+m, q+2 \sigma-2, h}}^{2} .
$$

Noting that $T_{j k}\left(a_{i}\right)=T_{j k}\left(b_{i}\right)+T_{j k}\left(c_{i}\right)+T_{j k}\left(d_{i}\right)$, we have by Lemma 2.15

$$
\begin{aligned}
\sum_{j, k}\left\|T_{j k}\left(a_{i}\right) D_{0,1}^{m-i} u\right\|_{W_{l, q, h}}^{2} & \leqslant C_{l, q}\left\{\left[b_{i}\right]_{M_{2}(l, q)}^{(i, \mu)}+\left[c_{i}\right]_{M_{2}(l, q)}^{(i)}+\left|d_{i}\right|_{\mathcal{M}_{2}(l, q)}^{(i)}\right\}^{2} \\
& \times\|u\|_{W_{l+m, q-1},}^{2} .
\end{aligned}
$$


Thus we obtain

$$
R_{j k}(a)=\tilde{S}_{j k}(x, D) \varphi_{j k}\left(x, D^{\prime}\right)+\tilde{T}_{j k}(a)(x, D),
$$

which satisfies (2.22).

\section{$\S 3$. Quasilinear case}

We at first consider a following quasilinear equation of unknown $u(x)$,

$$
\left\{\begin{array}{l}
p\left(x, D^{m-1} u, D\right) u(x)=b\left(x, D^{m-1} u\right), \\
\left(\partial / \partial x_{0}\right)^{j} u\left(0, x^{\prime}\right)=0, \quad j=0,1, \cdots, m-1,
\end{array}\right.
$$

where $p(x, y, \xi)$ is a hyperbolic polynomial with respect to $\xi_{0}$. Here we assume that the coefficients $a_{\alpha}(x, y)$ of $p(x, y, \xi)$ satisfy $(0.4)$ for $(x, y)$ in $G \times V$. Let $\left.\theta(x)=\theta_{0}(x), \cdots, \theta_{n}(x)\right)$ be a mapping of $R^{n+1}$ to $G$ such that

$$
\theta(x)= \begin{cases}x & \text { for } \quad|x| \leqslant R_{1} / 2 \\ 0 & \text { for } \quad|x| \geqslant R_{1}\end{cases}
$$

and each $\theta_{i}(x)(i=0, \cdots, n)$ be in $\gamma_{A_{0}}^{(s)}\left(R^{n+1}\right)$, and $\rho(x)$ be a function in $C_{0}^{\infty}\left(R^{n+1}\right)$ such that $\int \rho d x=1$ and $\chi(x) \in \gamma_{A_{0}}^{(s)}\left(R^{n+1}\right)$ such that $\chi(x)=1$ on $|x| \leqslant R_{1} / 2, \chi(x)=0$ for $|x| \geq R_{1}$. We put

$$
\begin{aligned}
p_{\iota}\left(x, D^{m-1} u, D\right) & =p\left(\theta(x), \rho_{\iota} *\left(\chi D^{m-1} u\right), D\right) \\
& =\sum_{|\alpha|=m} a_{\alpha}\left(\theta(x), \rho_{\iota} *\left(\chi D^{m-1} u\right)\right) D^{\alpha},
\end{aligned}
$$

where $\rho_{\varepsilon}(x)=\varepsilon^{-(n+1)} \rho\left(\varepsilon^{-1} x\right)$. Then, if $u$ is in $W_{\hat{\imath}, \hat{q}, h}^{(\Lambda)}$, where $\Lambda=\left(T-x_{0}\right)(h+$ $\left.\left\langle D^{\prime}\right\rangle_{h}^{*}\right), \kappa=s^{-1}, 0 \leq T \leq \min \left(\left(24 n^{\kappa} A_{0}^{\kappa}\right)^{-1}, R_{1}\right)$, then $\rho_{\iota} *\left(\chi D^{m-1} u\right)$ in $W_{l-m+1, q, h}^{(A)}$ for any $l, q$ in $R^{1}$ and $\varepsilon>0$, and satisfies for $l$ and $\hat{q}$,

$$
\left\|\rho_{\ell} * \chi D^{m-1} u\right\|_{W_{\hat{l}-(A)+1, \hat{q}, h} \leqslant C_{0}\|u\|_{W_{\hat{l}, \hat{q}, h}^{(A)}}} .
$$

where $C_{0}$ is independent of $h$ and $\varepsilon$. Therefore it follows from Proposition 2.9 that if $l \geqslant n+3, \hat{q} \geq 0$ and $u$ is in $W_{\hat{l}, \hat{q}, h}^{(1)}$ satisfies

$$
\|u\|_{w_{\hat{\imath}, \hat{q}, h}^{(\Lambda)}} \leqslant\left(2 r A_{0} C_{0}\right)^{-1},
$$

then $a_{\alpha}\left(\theta(x), \rho_{\varepsilon} *\left(\chi D^{m-1} u\right)-a_{\alpha}(\theta(x), 0)\right.$ is in $W_{\hat{\imath}-m+1, \hat{q}, h}^{(1)}$ and

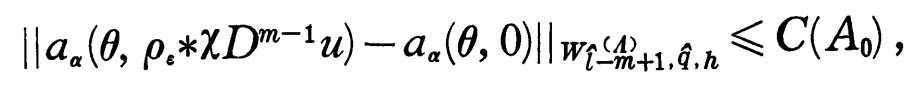

where $\Lambda=\left(T-x_{0}\right)\left(h+\left\langle D^{\prime}\right\rangle_{h}^{\star}\right), \kappa=s^{-1}$ and

$$
0 \leqslant T \leqslant \min \left(\left(24 n^{*} A_{0}^{*}\right)^{-1}, R_{1}\right) .
$$

Moreover for $l>\hat{l}$ or $q>\hat{q}$, we have 
(3.4)。

$$
\left\|a_{\alpha}\left(\theta, \rho_{\varepsilon} * \chi D^{m-1} u\right)-a_{\alpha}(\theta, 0)\right\|_{W_{l-m+1, q, h}^{(1)}} \leqslant C\left(A_{0}, \varepsilon\right),
$$

for $\varepsilon>0$, where $C(A, \varepsilon)$ depends on $\varepsilon$ but does not on $h$.

Now, instead of (3.1) we consider the Cauchy problem for $p_{c}\left(x, D^{m-1} u, D\right)$ as follows,

$$
\left\{\begin{array}{l}
p_{c}\left(x, D^{m-1} u, D\right) v(x)=f(x), x \in[0, T] \times R^{n}, \\
\left(\partial / \partial x_{0}\right)^{j} u\left(0, x^{\prime}\right)=0, \quad j=0, \cdots, m-1 .
\end{array}\right.
$$

If $u(x)$ is given, then (3.6) is an equation for $v(x)$. Assume that $u(x)$ in $W_{\hat{l}, \hat{q}, h}^{(1)}$ satisfies (3.3), where $\Lambda=\left(\mathrm{T}-\mathrm{x}_{0}\right)\left(h+\left\langle D^{\prime}\right\rangle_{h}^{c}\right)$ satisfies (3.5), and $\hat{l}$ is a large integer and $\hat{q} \geq 0$, fixed, and that the coefficients $a_{\alpha}(x, y)$ satisfy $(0.4)$ and $p(x, y, \xi)$ is hyperbolic with respect to $\xi_{0}$. Then $a_{s}(x, \xi)=p_{s}\left(x, D^{m-1} u, \xi\right)$ is also hyperbolic with respect to $\xi_{0}$, and it follows from Lemma 1.4 that there exists a positive constant $C=C\left(\|u\|_{W_{\hat{l}, \hat{q}, h}^{(1)}}\right)$ such that

$$
\begin{aligned}
& \left|a_{\varepsilon}\left(x, \xi_{A}\right)^{-1}\right| \leqslant C\langle\xi\rangle_{h}^{-m}\left\langle\xi^{\prime}\right\rangle_{h}^{\tau}, \\
& \left|\frac{a_{s}^{(\alpha)}\left(x, \xi_{A}\right)}{a_{\varepsilon}\left(x, \xi_{A}\right)}\right| \leqslant C\left\langle\xi^{\prime}\right\rangle_{h}^{-\kappa} \\
& \left|\frac{a_{\varepsilon(\alpha)}\left(x, \xi_{A}\right)}{\alpha_{\varepsilon}\left(x, \xi_{A}\right)}\right| \leqslant C\left\langle\xi^{\prime}\right\rangle_{h}^{1-\kappa},
\end{aligned}
$$

for $|\alpha|=1, x \in R^{n+1}, \xi_{1}=\left(\xi_{0}-i\left(h+\left\langle\xi^{\prime}\right\rangle_{h}^{\kappa}\right), \xi^{\prime}\right), \xi \in R^{n+1}$, where $\kappa=s^{-1}, \tau=\nu(1-\kappa)$ and $\nu$ is the maximal multiplicity of roots of $a_{c}$.

THEOREM 3.1. Assume that the above conditions for $p$ are valid and $\hat{l}$ is a large integer and $\hat{q} \geq \tau$. Then for any $l, q$ in $R^{1}$ and $\varepsilon>0$ there exist positive numbers $h=h(l, q, \varepsilon)$ and $C=C(l, q, \varepsilon)$ such that for $u$ in $W_{\hat{\imath}, \hat{q}, h}^{(1)}$ satisfying (3. 3) and any $v$ in $W_{l, q, h}^{(1)}$

$$
\|v\|_{W_{l, q, h}^{(A)}} \leqslant C(l, q, \varepsilon)\left\|p_{\mathbf{c}}\left(\cdot, D^{m-1} u, D\right) v\right\|_{W_{l-m, q+r, h}^{(A)}}
$$

where $\Lambda=\left(T-x_{0}\right)\left(h+\left\langle D^{\prime}\right\rangle_{h}^{*}\right), \quad \kappa=s^{-1}, \quad 0<s<\nu(\nu-1)^{-1}$ and $T$ satisfies (3.5). In paticular if $0 \leq l \leq \hat{l}+1$ and $0 \leq q \leq \hat{q}-\tau$, then $h(l, q, \varepsilon)$ and $C(l, q, \varepsilon)$ do not depend on $\varepsilon$.

For the adjoint operator $p_{c}^{(*)}$ of $p_{s}$, we obtain analogously,

THEOREM 3.2. Assume that the conditions of Theorem 3.1 are valid. Then for any $l, q$ in $R^{1}$ and $\varepsilon>0$ there exist $h=h(l, q, \varepsilon)$ and $C=C(l, q, \varepsilon)$ such that for $u$ in $W_{l, q, h}^{(A)}$ satisfying (3.3) and for any w in $W_{l, q, h}^{(-A)}$,

$$
\|w\|_{W_{l, q, h}^{(-,)}} \leq C(l, q, \varepsilon)\left\|p_{\bullet}^{(*)}\left(\cdot, D^{m-1} u, D\right) w\right\|_{w_{l=m, q+\tau, h}^{(-1)}} .
$$


Therefore it follows from that Theorem 3.1 and Theorem 3.2 that by virtue of Riesz' theorem we obtain an existence theorem (c. f. [4]) as follows,

Theorem 3.3. Assume that the conditions of Theorem 3.1 are valid. Then if $0 \leqslant l \leq \hat{l}+1$ and $0 \leq q<\hat{q}-\tau$, there exist $h=h(l, q)$ and $C=C(l, q)$ such that for any $u$ in $W_{\hat{\imath}, \hat{q}, h}^{(1)}$ satisfying (3.3) and for any $f$ in $W_{l-m, q+\varepsilon, h}^{(1)}$, we have a unique solution $v$ in $W_{l, q, h}^{(1)}$ of the following equation,

$$
p\left(\theta(x), \chi D^{m-1} u(x), D\right) v(x)=f(x), \quad x \text { in } R^{n+1},
$$

and $v$ satisfies

$$
\|v\|_{W_{l, q, h}^{(A, h}} \leqslant C(l, q)\|f\|_{W_{l-m, q+r, h}^{(A)}} .
$$

Since the conditions of Theorem 3.3 are invariant under Holmgen's transforms, the solution of (3.11) with zero initial data has a finite propagation speed. Therefore we obtain the following (c. f. Theorem 4.5 in [4]),

THeOREM 3.4. Assume that the conditions of Theorem 3.1 are valid. If the support of $f$ is compact in $[0, \infty) \times R^{n}$, then the solution $v$ of (3.11) has a compact support in $\left[0, T_{0}\right] \times R^{n}=\Omega_{T_{0}}$ for $T_{0}>0$ and satisfies

$$
\|\boldsymbol{v}\|_{W_{l+1, q-\tau, h}^{(l)}\left(\Omega_{T_{0}}\right)} \leqslant C(l, q)\|f\|_{W_{l-m+1, q, h}^{(\Lambda)}\left(\Omega_{T_{0}}\right)},
$$

for $2 m+(n+1) / 2<l \leqslant \hat{l}$ and $\tau \leqslant q \leqslant \hat{q}$.

Proof of Theorem 3.1. We put

$$
\begin{aligned}
& a_{s}(x, D)=p_{c}\left(x, D^{m-1} u, D\right) \\
& g=e^{-\Lambda} a_{\varepsilon}(x, D) v=a_{s \Lambda}(x, D) w, \quad w=e^{4} v, \\
& a_{s \Lambda}(x, D)=e^{4} a_{s} e^{-\Lambda}, \quad a_{s j k}(D)=a_{s}\left(x_{j k}(D), D_{\Lambda}\right) \\
& D_{4}=e^{\Lambda} D e^{-4}=\left(D_{0}-i\left(h+\left\langle D^{\prime}\right\rangle_{h}^{k}\right), D^{\prime}\right)
\end{aligned}
$$

where $x_{j k}(D)$ is given by (2.9). Then applying $\varphi_{j k}\left(x, D^{\prime}\right)$ to $g$ and noting that $e^{\Lambda} a_{\imath}\left(x_{j k}, D\right) e^{-1}=a_{s}\left(x_{j k}, D_{A}\right)=a_{s j k}(D)$,

$$
\begin{aligned}
& a_{s j k}(D) \varphi_{j k}\left(x, D^{\prime}\right) w=\left(a_{s j k}(D)-a_{s \Lambda}(x, D)\right) \varphi_{j k} w+\left[a_{s 1}, \varphi_{j k}\right] w \\
& \quad+\varphi_{j k}\left(x, D^{\prime}\right) g(x),
\end{aligned}
$$

where [ , ] stands for a commutator. Since $a_{\imath j k}(\xi)=a_{\imath}\left(x_{j k}\left(\xi^{\prime}\right), \xi_{1}\right)$ satisfies (3.7), there exists the inverse $a_{s j k}(D)^{-1}$ of $a_{s j k}(D)$ in $W_{l, q, k}$. Hence from (2.21) we have 


$$
\begin{aligned}
\varphi_{j k} w & =a_{\varepsilon j k}(D)^{-1}\left[\sum _ { | \alpha | = 1 } \left\{a_{\varepsilon(\alpha)}\left(x_{j k}, D_{A}\right)\left(x_{A}-x_{j k}\right)^{\alpha} \varphi_{j k} w\right.\right. \\
& +\sum_{|\alpha|=1}\left\{a_{s}^{(\alpha)}\left(x_{j k}, D_{A}\right)+\sum_{|\beta|=1} a_{\varepsilon(\beta)}\left(x_{j k}, D_{A}\right) x_{j k}^{\beta(\alpha)}\right\} \varphi_{j k} w \\
& \left.+R_{j k}\left(a_{s}\right)(x, D) w+\varphi_{j k}\left(x, D^{\prime}\right) g(x)\right]
\end{aligned}
$$

Since for $|\alpha|=1 \quad a_{s}\left(x_{j k}\left(\xi^{\prime}\right), \xi_{A}\right)^{-1} a_{s}^{(\alpha)}\left(x_{j k}\left(\xi^{\prime}\right), \xi_{A}\right)$ and $a_{\varepsilon}\left(x_{j k}\left(\xi^{\prime}\right), \xi_{A}\right)^{-1} a_{\varepsilon(\alpha)}\left(x_{j k}\left(\xi^{\prime}\right), \xi_{A}\right)$ satisfy (3.8) and (3.9) respectively, we obtain

$$
\begin{aligned}
& \left\|\varphi_{j k} w\right\|_{W_{l, q, h}}^{2} \leqslant C\left[\sum_{|\alpha|=1}\left\|\left(x_{1}-x_{j k}\right)^{\alpha} \varphi_{j k} w\right\|_{W_{l, q+1-\kappa, h}}^{2}\right. \\
& \quad+\sum_{|\alpha|=1}\left\{\left\|\varphi_{j k(\alpha)} w\right\|_{W_{l, q-\kappa, h}}^{2}+\sum_{|\beta|=1}\left\|x_{j k}^{\beta(\alpha)} \varphi_{j k(\alpha)} w\right\|_{W_{l, q+1-\kappa}, h}^{2}\right\} \\
& \left.\quad+\left\|R_{j k}\left(a_{\varepsilon}\right) w\right\|_{W_{l-m, q+\tau, h}}^{2}+\left\|\varphi_{j k} g\right\|_{W_{l-m, q+\tau}, h}^{2}\right]
\end{aligned}
$$

where $C$ is independent of $h$. Noting that $\left(x_{j k}^{\beta}\right)^{(\alpha)}=(\partial / \partial \xi)^{\alpha}\left(x_{j k}\left(\xi^{\prime}\right)^{\beta}\right)$ is in $S_{1,0, h}^{(0,-|\alpha|)}$ uniformly in $(j, k)$, we obtain from Proposition 2.12,

$$
\begin{gathered}
\sum_{j, k}\left\|\varphi_{j k} w\right\|_{W_{l, q, h}}^{2} \leqslant C\left[\|w\|_{W_{l, q+1-\kappa-\sigma, h}}^{2}+\|w\|_{W_{l, q-\kappa+\sigma, h}}^{2}+\|w\|_{W_{l, q-\kappa, h}}^{2}\right. \\
\left.+\sum_{j, k}\left\|R_{j k}\left(a_{\varepsilon}\right) w\right\|_{W_{l-m, q+\tau, h}}^{2}+\|g\|_{W_{l-m, q+\tau}, h}^{2}\right]
\end{gathered}
$$

where $C$ is independent of $\varepsilon$ and $h$. We decompose

$$
\begin{aligned}
a_{\varepsilon}(x, D) & =p\left(\theta(x), \rho_{\varepsilon} * \chi D^{m-1} u, D\right) \\
& =p\left(\theta(x), \rho_{\varepsilon} * \chi D^{m-1} u, D\right)-p(\theta(x), 0, D) \\
& +p(\theta(x), 0, D)-p(0,0, D)+p(0,0, D) \\
& =b_{\varepsilon}(x, D)+c(x, D)+d(D) .
\end{aligned}
$$

Then we have

$$
b_{\varepsilon}(x, D)=\sum_{i=0}^{m} b_{\imath i}\left(x, D^{\prime}\right) D_{0}^{m-i}=\sum_{|\alpha|=m} b_{\varepsilon \alpha}(x) D^{\alpha},
$$

where $b_{\iota \alpha}(x)=a_{\alpha}\left(\theta(x), \rho_{\iota} * \chi D^{m-1} u\right)-a_{\alpha}(\theta(x), 0)$ satisfies (3.4) and (3.4). Hence $b_{s i}\left(x, \xi^{\prime}\right)$ is in $C_{0}^{\infty}\left([-T, T] ; \mathscr{A}^{(i, A)}\right)$ and satisfies

$$
\left[b_{\varepsilon i}\right]_{l}^{(i, \Lambda)} \leq C \sup _{\alpha}\left\|b_{\varepsilon \alpha}\right\|_{W_{l, 0, h}^{(A)}} .
$$

Noting that the coefficients of $c(x, D)$ and $d(D)$ do not depend on $\varepsilon$ and $u$, and that the coefficients $c_{i}\left(x, \xi^{\prime}\right)$ of $\xi_{0}^{m-i}$ in $c(x, \xi)$ is in $C_{0}^{k}\left([-T, T] ; \mathscr{A}_{s, A_{0}, h}^{(i)}\right)$, we obtain by virtue of (2.22) in Proposition 2.16, 


$$
\begin{aligned}
\sum_{j, k}\left\|R_{j k}\left(a_{\varepsilon}\right) w\right\|_{W_{l-m, q+\tau}, h}^{2} & \leq C\left[\operatorname { s u p } _ { 0 \leq i \leq m } \left\{\left[b_{\varepsilon i}\right]_{M_{2}(l-m, q+\tau)}^{(i)}+\left|c_{i}\right|_{s, A_{0}, M_{2}(l-m, q+\tau)}^{(i)}\right.\right. \\
& \left.\left.+\left|d_{i}\right|_{M_{1}(l-m, q+\tau)}^{(i)}\right\}\right]^{2}\|w\|_{W_{l, q+\tau-1, h}}^{2} .
\end{aligned}
$$

Thus if we take $\sigma(O<\sigma<1 / 2)$ such that

$$
\rho=\inf \{\kappa+\sigma-1, \kappa-\sigma, \kappa, 1-\tau\}>0,
$$

we have from (3.14), (3.15) and (3.4)

$$
\|w\|_{W_{l, q, h}}^{2} \leqslant C_{l, q}\left(1+C\left(\varepsilon, A_{0}\right)\right)^{2} h^{-2 \rho}\|w\|_{W_{l, q, h}}^{2}+C_{l, q}\|g\|_{W_{t-m, q+\tau}, h}^{2} .
$$

Hence if we choose $h$ such that

$$
C_{l, q}\left(1+C\left(\varepsilon, A_{0}\right)\right) h^{-2 \rho} \leqslant 1 / 2,
$$

we obtain the estimate (3.10).

Next we shall prove (3.10) for a positive integer $l$. Applying $D^{\alpha}(|\alpha|=l)$ to $g$, we have

$$
a_{\varepsilon \Lambda}(x, D) D^{\alpha} w=\left[a_{\varepsilon \Lambda}(x, D), D^{\alpha}\right] w+D^{\alpha} g=g_{\alpha} .
$$

Then by virtue of (3.14) and (3.15) we have

$$
\begin{aligned}
\left\|D^{\alpha} w\right\|_{W_{0, q, h}}^{2} & \leqslant C_{q}\left(1+\sup _{\beta}\left\|b_{\epsilon \beta}\right\|_{W_{M_{2}(-m, q), 0, h}^{(\alpha)}}\right)^{2} h^{-2 \rho}\left\|D^{\alpha} w\right\|_{W_{0, q, h}}^{2} \\
& +C_{q}\left\|g_{\alpha}\right\|_{W_{-m, q+\tau, h}}^{2},
\end{aligned}
$$

where $C_{q}$ is independent of $\varepsilon$ and $h$. For $g_{\alpha}$ we have

$$
\left\|g_{\alpha}\right\|_{W_{-m, q+\tau, h}}^{2} \leqslant\|g\|_{W_{l-m, q+\tau, h}}^{2}+\left\|\left[a_{\varepsilon \Lambda}, D^{\alpha}\right] w\right\|_{W_{-m, q+\tau, h}}^{2},
$$

and

$$
\left\|\left[a_{\varepsilon, 1}, D^{\alpha}\right] w\right\|_{W_{-m, q+\tau, h}} \leqslant\left\|\left[b_{\varepsilon \Lambda}, D^{\alpha}\right] w\right\|_{W_{-m, q+\tau}, h}+\left\|\left[c_{\Lambda}, D^{\alpha}\right] w\right\|_{W_{-m, q+\tau, h}} .
$$

Since $\left(c_{A}\right)(x, \xi)$ is in $S_{1,0, h}^{(m, 0)}$, we have from Proposition 2.6,

$$
\left\|\left[c_{A}, D^{\alpha}\right] w\right\|_{W_{-m, q+\tau}, h} \leqslant C\|w\|_{W_{l-1, q+\tau, h}} \leqslant C h^{-\rho}\|w\|_{w_{l, q, h}},
$$

where $C$ is independent of $\varepsilon$ and $h$.

Finally we shall estimate $\left[b_{\varepsilon,}, D^{\alpha}\right] w$. We have 


$$
\begin{aligned}
{\left[b_{\epsilon \Lambda}, D^{\alpha}\right] w } & =-\sum_{0 \neq \gamma \leqslant \alpha}\left(\begin{array}{l}
\alpha \\
\gamma
\end{array}\right) b_{\varepsilon \Lambda(\gamma)}(x, D) D^{\alpha-r} w \\
& =-\sum_{\gamma}\left(\begin{array}{c}
\alpha \\
\gamma
\end{array}\right) \sum_{|\beta|=m} b_{\epsilon \beta \Lambda(\gamma)}(x) D_{\Lambda}^{\beta} D^{\alpha-r} w \\
& =-\sum_{|\beta|=m} \sum_{0<\gamma \leqslant \alpha}\left(\begin{array}{l}
\alpha \\
\gamma
\end{array}\right) e^{\Lambda}\left(D_{-\Lambda}^{r} b_{\varepsilon \beta}\right) D^{\beta} D_{-\Lambda}^{\alpha-\gamma} v+D^{\beta} v D_{-\Lambda}^{\alpha} b_{\iota \beta},
\end{aligned}
$$

where $D_{-4}=e^{-\Lambda} D e^{\Lambda}$. Hence we have

$$
\left\|\left[b_{\iota s}, D^{\alpha}\right] w\right\|_{W_{-m, q+\tau, h}} \leqslant C \sum_{0<\tau \leqslant \alpha,|\beta|=m}\left\|\left(D_{-\Lambda}^{r} b_{\epsilon \beta}\right)\left(D^{\beta} D_{-\Lambda}^{\alpha-\gamma} \boldsymbol{v}\right)\right\|_{W_{-m, q+\tau, h}^{(A)}} .
$$

For $0<|\gamma| \leqslant l_{0}$ (determined later on), we obtain by virtue of Proposition 2.7,

$$
\begin{aligned}
& \left\|\left(D_{-1}^{r} b_{\iota \beta}\right)\left(D^{\beta} D_{-\Lambda}^{\alpha-\gamma} v\right)\right\|_{W_{-m, q+\tau, h}^{(A)}} \\
& \leqslant C\left\|D_{-\Lambda}^{r} b_{\varepsilon \beta}\right\|_{\left.W_{M_{A}(1)}^{(A)}, q+\tau\right), q+\tau, h}\|v\|_{W_{l-1, q+\tau, h}^{(\Lambda)}}
\end{aligned}
$$

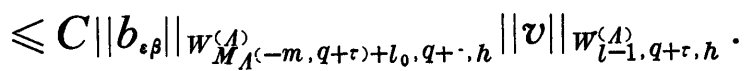

For $|\gamma|>l_{0}$, we have analogously,

$$
\begin{aligned}
& \left\|\left(D_{-\Lambda}^{\gamma} b_{\varepsilon \beta}\right)\left(D^{\beta} D_{-\Lambda}^{\alpha-\gamma} v\right)\right\|_{W_{-m, q+, h}^{(\alpha)}} \\
& \leqslant C\left\|D^{\beta} D_{-\Lambda}^{\alpha-\tau} v\right\|_{W_{M_{\Lambda}^{(-m, q+\tau), q+\tau, h}}^{(\mathcal{L})}}\left\|b_{\varepsilon \beta}\right\|_{W_{l-m, q+\tau, h}^{(\lambda)}}
\end{aligned}
$$

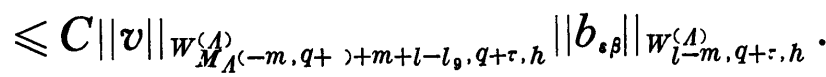

Therefore if $\hat{l} \geqslant 2 M_{1}(-m, \hat{q})+m$, we can choose $l_{0}$ such that

$$
\begin{aligned}
& M_{\Lambda}(-m, \hat{q})+l_{0} \leqslant \hat{l}-m+1, \\
& M_{\Lambda}(-m, \hat{q})+m-l_{0} \leqslant-1,
\end{aligned}
$$

and we have from (3.4) for $|\alpha|=l \leqslant \hat{l}+1$ and $q+\tau \leqslant \hat{q}$,

$$
\left\|\left[b_{\varepsilon \Lambda}, D^{\alpha}\right] w\right\|_{W_{-m, q+\tau}, h} \leqslant C\|v\|_{W_{l-1, q+\tau, h}^{(A)}} \leqslant C h^{-\rho}\|w\|_{w_{l, q, h}^{(A)}},
$$

where $C$ is independent of $h$ and $\varepsilon$. Thus we obtain from (316,) (3.17), (3. 18) and (3.19),

$$
\begin{aligned}
\|w\|_{w_{l, q, h}} & \leqslant C h^{-\rho}\left\{1+\sup \left\|b_{\varepsilon \beta}\right\|_{W_{M_{2}(-m, q), 0, h}^{(q)}}\right\}\|w\|_{W_{l, q, h}} \\
& +C\|g\|_{W_{l-m, q+\tau}, h},
\end{aligned}
$$

where $C$ is independent of $h$ and $\varepsilon$. If $\hat{l}-m+1 \geqslant M_{2}(-m, \hat{q})$, then we have by (3.4) and (3.20)

$$
\|v\|_{W_{l, q, h}^{(A)}} \leqslant C\|f\|_{W_{l-m, q+\tau, h}^{(A)}}
$$


for any $h \geq h_{0}$, where $C$ and $h_{0}$ are independent of $\varepsilon$. Thus we have proved Theorem 3.1.

Now we return to the equation (3.1). By Taylor's expantion, we put

$$
\tilde{u}(x)=u(x)-\sum_{j=0}^{M}\left(\partial / \partial x_{0}\right)^{j} u\left(0, x^{\prime}\right) x_{0}{ }^{j}(j !)^{-1} .
$$

Then we can regard (3.1) the equation of $\tilde{u}(x)$ and if we take $M$ sufficiently large, we may assume without loss of generality

$$
b\left(x, D^{m-1} u\right)=x_{0}^{\hat{\imath}-m+1} \tilde{b}\left(x, D^{m-1} u\right) .
$$

Let $\chi(x)$ be in $\gamma_{A_{0}}^{(s)}\left(R^{n+1}\right)$ such that $\chi=1$ for $|x| \leqslant R_{1} / 2$ and $\chi=0$ for $|x| \geq R_{1}$. Then if $u$ is in $W_{\hat{l}, \hat{q}, h}^{(\alpha)}$ satisfying (3.3), it follows from Lemma 1.3 and Proposition 2.9 that $\chi \tilde{b}\left(x, D^{m-1} u\right)$ is in $W_{\hat{l}-m+1, \hat{q}, h}^{(1)}$. We denote by $B\left(T_{0}, R\right)$ all functions $u(x)$ in $W_{\hat{l}, \hat{q}, h}^{(\hat{d})}$ satisfying

$$
\|u\|_{w_{\hat{i}, \hat{q}, h}^{(\Lambda)}} \leqslant\left(2 r A_{0} C_{0}\right)^{-1},
$$

and

$$
\{\text { suppu }\} \cap\left\{x_{0} \leqslant T_{0}\right\} \subset\left[0, T_{0}\right] \times\left\{\left|x^{\prime}\right| \leqslant R\right\} .
$$

We define an operator $K$ of $B\left(T_{0}, R\right)$ to $B\left(T_{0}, R\right)$ such that for $K(u)$ is the solution of the following equation,

$$
\left\{\begin{array}{l}
p\left(\theta(x), D^{m-1} u, D\right) v(x)=\chi(x) b\left(x, D_{u}^{m-1}\right), \quad x_{0}>0, \\
D_{0}^{j} v\left(0, x^{\prime}\right)=0, \quad j=0, \cdots, m-1 .
\end{array}\right.
$$

Then it follows from (3.12) in Theorem 3. 4 that

$$
\begin{aligned}
\|K(u)\|_{W_{\hat{l}, \hat{q}, h\left(u_{T_{0}}\right)}^{(A)}} \leqslant C\|x b\|_{W_{\hat{l}-m, \hat{q}+\tau, h}^{(1)}\left(s_{T_{0}}\right)} \\
\leqslant C_{1}\left\|x_{0}^{-m+1} \tilde{b}\right\|_{W_{\hat{l}-m, \hat{q}+\tau, h}^{(A)}\left(s_{T_{0}}\right)} \\
\leqslant C_{2} T_{0}\|\tilde{b}\|_{W_{\hat{l}-m, \hat{q}+\tau, h}^{(A)}\left(\Omega_{T_{0}}\right)} \\
\leqslant C_{3} T_{0} \leq\left(2 r A_{0} C_{0}\right)^{-1},
\end{aligned}
$$

if we choose $T_{0}$ sufficiently small, and that also $K(u)$ is in $W_{\hat{\imath}+1, \hat{q}-\tau, h}^{(A)}\left(\Omega_{T_{0}}\right)$ and $\left\{K(u) ; u \in B\left(T_{0}, R\right)\right\}$ is a bounded set in $W_{\hat{\imath}+1, \hat{q}-\tau, h}^{(A)}\left(\Omega_{T_{0}}\right)$. Moreover if we take $R$ suitably, it is clearly that $K(u)$ has the property (3.23). Since $\tau<1$, it follows from Relleich's theorem that the image $\left\{K(u) ; u \in B\left(T_{0} R\right)\right\}$ is compact in $W_{\hat{\imath}, \hat{q}, h}^{(\lambda)}\left(\Omega_{T_{0}}\right)$. Therefore by applying Schauder's fixed point theorem, we know that $K$ has a fixed point in $B\left(T_{0}, R\right)$. Thus we have, 
Theorem 3.5. Assume that the condition of Theorem 3.1 are valid. Then for any large integer $l\left(l>M_{2}(-m, \tau)\right)$ there exists a positive number $h$ such that there is a function $u(x)$ in $W_{l, r, h}^{(A)}$ satisfying (3.1) in a neighborhood of 0 in $R^{n+1}$.

\section{$\S 4$. Proof of Theorem 0.1}

We shall reduce the nonlinear equations $(0.1)$ to the quasilinear ones following Dionne [3].

We put

$$
p_{j k}(x, y, D)=\sum_{\alpha \in M_{j k}} F_{j y_{\alpha}}(x, y) D^{\alpha},
$$

and

$$
\begin{aligned}
& U=\left(U_{0}, U_{1}, \cdots, U_{n+1}\right), \quad U_{i}=\left(U_{1 i}, \cdots, U_{N i}\right), i=0,1, \cdots, n+1, \\
& U_{j i}=\partial / \partial x_{i} U_{j}, \quad j=1, \cdots, N, i=0,1, \cdots, n, \\
& U_{j n+1}=u_{j}, \quad j=1, \cdots, N .
\end{aligned}
$$

Differentiating $(0.1)$ by $x_{i}$,

$$
\begin{gathered}
\sum_{k=1}^{N} p_{j k}\left(x, D^{M_{j}} u, D\right) U_{k i}+F_{x_{i}}\left(x, D^{M_{j}} u\right)=0, \\
j=1, \cdots, N, \quad i=0, \cdots, n .
\end{gathered}
$$

By Taylor's expansion,

$$
\begin{gathered}
F_{j}\left(x, D^{M_{j}} u\right)-F(x, 0)=\sum_{k=1}^{N} p_{j k}\left(x, D^{M_{j}} u, D\right) U_{k n+1} \\
+G_{j}\left(x, D^{M_{j}} u\right), \quad j=1, \cdots, N,
\end{gathered}
$$

where $G_{j}(x, y)=\int_{0}^{1} \sum_{i=1}^{N} \sum_{\alpha \in M_{j i}} F_{i y_{\alpha}}(x, \theta y) y_{\alpha} d \theta$.

Here we can assume without loss of generality that

$$
m+n_{k}-n_{j} \geq 1, \quad j, k=1, \cdots, N .
$$

In fact, if it is not so, we choose a positive integer $m^{\prime}$ such that $m+m^{\prime}+$ $n_{k}-n_{j} \geqslant 1$ for all $j, k$. Then we operate a strictly hyperbolic operator $p_{j}(D)$ of order $m^{\prime}$ to $F_{j}=0$,

$$
p_{j}(D) F_{j}\left(x, D^{M_{j}} u\right)=\widetilde{F}_{j}\left(x, D^{\widetilde{\varkappa_{j}}} u\right)=0, \quad j=1, \cdots, N .
$$

Then it is clearly that

$$
|\alpha| \leqslant m+m^{\prime}+n_{k}-n_{j} \text { for } \alpha \in \widetilde{M}_{j k} .
$$

and the characteristic polynomial $\tilde{p}(x, y, \xi)$ for $\left\{\tilde{F}_{j}\right\}$ is 


$$
\tilde{p}(x, y, \xi)=\prod_{j=1}^{N} p_{j}(\xi) p(x, y, \xi),
$$

of which maximal multiplicity is same to one of $p(x, y, \xi)$, if we choose $p_{j}(\xi)$ suitably. It is evident that the equations $(0.1)$ are equivalent to (4.5).

Let $e_{l}=(0, \cdots, 0,0, \hat{1}, 0, \cdots, 0)$ be a unit vector in $R^{n+1}$. We put

$$
\begin{aligned}
& l(\alpha)=\inf \left(l ; \alpha_{l} \neq 0\right) \quad \text { for } \quad \alpha \neq 0, \\
& r(\alpha)=e_{l(\alpha)} \quad \text { for } \quad \alpha \neq 0 .
\end{aligned}
$$

For $\alpha \in M_{j k}$, we define

$$
\beta(\alpha)=\alpha-\gamma(\alpha), \alpha \in M_{j k},|\alpha|=m+n_{k}-n_{j} .
$$

Then we can rewrite

$$
D^{\boldsymbol{M}_{j k}} u_{k}=\left\{D^{L_{j k}^{i}} U_{k i}, i=0, \cdots, n+1\right\}, \quad j, k=1, \cdots, N
$$

where

$$
L_{j k}^{i}=\left\{\begin{array}{rr}
\left\{\beta \in M_{j k} ; \exists \alpha \in M_{j k},|\alpha|=m+n_{k}-n_{j},\right. \text { s.t. } \\
\beta=\beta(\alpha), i=l(\alpha)\}, \quad i=0, \cdots, n, \\
\left\{\beta \in M_{j k} ;|\beta|<m+n_{k}-n_{j}\right\}, \quad i=n+1 .
\end{array}\right.
$$

Then we have evidently

$$
|\beta| \leqslant m-1+n_{k}-n_{j}, \quad \beta \in L_{j k}^{i}, \text { for } i=0,1, \cdots, n+1, j, k=1, \cdots, N .
$$

We define

$$
D^{L_{j}} U=\left\{D^{L_{j k}} U_{k i} ; i=0, \cdots, n+1, k=1, \cdots, N\right\} .
$$

Now we obtain the following equations,

$$
\left\{\begin{aligned}
\sum_{k=1}^{N} p_{j k}\left(x, D^{L_{j}} U, D\right) U_{k i} & =B_{j i}\left(x, D^{L_{j}} U\right), \\
j & =1, \cdots, N, \quad i=0,1, \cdots, n+1 . \\
D_{0}^{t} U_{k i}\left(0, x^{\prime}\right)=0, & t=0, \cdots, m-1, \quad i=0, \cdots, n+1,
\end{aligned}\right.
$$

where we may assume that the initial data are zero and $B_{j i}$ satisfies

$$
B_{j i}(x, y)=x_{0}^{\hat{l}-m+n_{j}} \tilde{B}_{j i}(x, y) .
$$

Moreover we assume that the coefficients of $p_{j k}(x, y, \xi)$ and $\tilde{B}_{j i}(x, y)$ satisfy (0.4). Then we consider the linearized equations of (4.7) as follows

$$
\sum_{k=1}^{N} p_{j k}\left(\theta(x), \chi D^{L_{j}} U, D\right) V_{k i}=\chi B_{j i}\left(x, D^{L_{i}} U\right),
$$


where $\theta(x)$ and $\chi(x)$ are given in $\S 3$.

THEOREM 4.1. Assume that $\hat{l}$ is a large integer, $\hat{q} \geq \tau$, and $U_{j i}(x)$ is in $W_{\hat{l}, \hat{q}, h}^{\hat{\imath},}$ satisfying

$$
\left\{\sum_{j, i}|| U_{j i} \|_{W_{\hat{l}+n_{j}, \hat{q}, h}^{(A)}}^{2}\right\}^{1 / 2} \leqslant\left(2 r C_{0} A_{0}\right)^{-1} .
$$

Then the following energy estimates hold

$$
\left.\sum_{j, i}|| V_{j i}\right|_{W_{l+n_{j}}, q, h} ^{2} \leqslant C_{l, q} \sum_{j, i} \|\left.\sum_{k=1}^{N} p_{j k} V_{k i}\right|_{W_{l}^{(1) m+n} j_{j}, q+r, h} ^{2}
$$

for $2 m N+(n+1) / 2+1<l \leqslant \hat{l}+1,0<q<\hat{q}-\tau$.

Proof. We put

$$
f_{j i}=\sum_{k=1}^{N} p_{j k} V_{k i} .
$$

Then we have for $|\alpha|=l$,

$$
\sum_{k=1}^{N} p_{j k}\left(D^{\alpha} V_{k i}\right)=-\sum_{k}\left[p_{j k}, D^{\alpha}\right] V_{k i}+D^{\alpha} f_{j i}=\tilde{f}_{j i} .
$$

It follows from (4.10) and Proposition 2.9 that the coefficients of $\left(p_{j k}(x\right.$, $\left.\left.D^{L_{j}} U, D\right)-p_{j k}(x, 0, D)\right)$ are in $W_{\hat{\imath}-m+1-n_{j}, \hat{q}, h}^{(\Lambda)}$. Hence we have by Lemma 1.2,

$$
\left\|\sum_{k}\left[p_{j k}, D^{\alpha}\right] V_{k i}\right\|_{W_{n^{-}}^{(h)}, q+\tau, h}^{2} \leqslant C \sum_{k}\left\|V_{k i}\right\|_{W_{l+n_{k-1}, q+\tau, h}^{(j)}}^{2} .
$$

We put

$$
D^{\alpha} V_{k i}=\sum_{t=1}^{N} H_{j t}\left(x, D^{L} U, D\right) W_{t},
$$

where $\left\{H_{k t}(x, y, \xi)\right\}$ is the cofactor matrix of $\left\{p_{j k}(x, y, \xi)\right\}$. Hence

$$
\begin{aligned}
\sum_{k} p_{j k}\left(x, D^{L_{j}} u, D\right) H_{k t}\left(x, D^{L} U, D\right) & =\delta_{j t} p\left(x, D^{L} U, D\right) \\
& +r_{j t}\left(x, D^{L} U, D\right),
\end{aligned}
$$

where $p(x, y, \xi)=\operatorname{det}\left\{p_{j k}(x, y, \xi)\right\}$ is hyperbolic in $\xi_{0}$ of order $m N$ and

(4.15) order $H_{j k} \leqslant m N-n_{k}+n_{j}-m$,

(4.16) order $r_{j k} \leqslant m N-n_{k}+n_{j}-1$.

Hence by (4.12) and (4.13)

$$
p\left(\theta(x), \chi D^{L} U, D\right) W_{j}=-\sum_{k} r_{j k} W_{k}+\tilde{f}_{j i} .
$$


Therefore by virtue of Theorem 3. 1, (4.13) and (4.16) we have

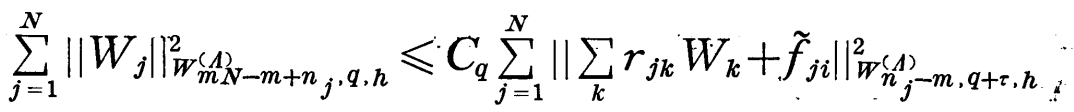

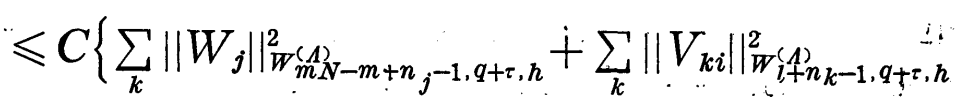

$$
\begin{aligned}
& \left.+\sum_{j}|| f_{j i} \|_{W_{l \neq i}\left\{n_{j}-m, q+\tau, h\right.}^{2}\right\} \text {, }
\end{aligned}
$$

where $C$ is independent of $h$. Since $\tau<1$, if $h$ is large, by (4.15) we have

$$
\begin{aligned}
& \sum_{j, k}\left\|\left.V_{j i}\right|_{W_{i, n_{j}, q, h}^{2}} ^{2} \leqslant \sum_{j, k}\right\| H_{j k} W_{k} \|_{W_{i \neq n_{j}, q, h}^{2}}^{2} \\
& \leqslant C \sum_{j}\left\|W_{j}\right\|_{W^{(1)} m N-m+n_{j}, q, h}^{2} \\
& \leqslant C_{l, q}\left\{\sum_{j, i}\left\|V_{j i}\right\|_{W_{i+n_{j}-1, q+\tau, h}^{2}}^{2}+\sum_{j, i}\left\|f_{j i}\right\|_{W^{\left(1-m+n_{j}\right.}, q+\tau, h}^{2}\right\},
\end{aligned}
$$

which implies (4.11).

It follows from Theorem 4, 1 that the linearized equations (4.9) have a solution $V=\left\{V_{j i}\right\}$ (c.f. Theorem 4.5 in [4]).

We define an operator $K$ as

$$
K(U)=V,
$$

and denote by $B\left(T_{0}, R\right)$ all functions $U(x)=\left\{U_{j i}\right\}, U_{j i}$ in $W_{\hat{\imath}+n_{j}, \hat{q}, h}^{(\lambda)}\left(\left[0, T_{0}\right] \times R^{n}\right)$ satisfying (4.10) and

$$
\operatorname{supp} U_{j i} \cap\left\{x_{0}<T_{0}\right\} \subset\left[0, T_{0}\right] \times\left\{\left|x^{\prime}\right| \leqslant R\right\} .
$$

Then $K$ is a compact operator in $B\left(T_{0}, R\right)$, if $T_{0}$ and $R$ are chosen suitably. Therefore (4.7) has a solution $U=\left\{U_{j i}\right\}$ and $\left\{U_{j n+1}\right\}$ satisfies the equations (0.4) in a neighborhood of 0 in $R^{n+1}$. Thus we have proved Theorem 0.1 .

\section{References}

[1] M. BRonshtein: Smoothness of roots of polynomials depending on parameters, Sibirski Math. Zh. 20 (1979) 493-501.

[2] M. BRonshtein: The parametrix of the Cauchy problem for hyperbolic operator with characteristics of variable multiplicity, Trudy Moscow Math. 41 (1980), 83-99.

[ 3 ] P. DionNe : Sur les problèmes de Cauchy hyperboliques bien posés, J. d'Analyse Math. 10 (1962), 1-90.

[4] K. KAJITANI : Cauchy problem for nonstrictly hyperbolic systems in Gevrey class, to appear in J. Math. Kyoto Univ.

[5] J. Kumanogo: Pseudo-differential operators, Iwanami, Tokyo (1975). 
[6] T. NISHITANI: Energy inequality for nonstrictly hyperbolic operators in the Gevrey class, to appear.

[7] J. LERAY et Y. OHYA: Systèmes nonlinèare hyperboliques nonstricts, Math. Ann. 70 (1967) 167-205.

[8] S. STEINBERG: Existence and uniqueness of solutions of hyperbolic equations which are not necessary strictly hyperbolic, J. Diff. Eq. 17 (1975), 119-153.

Institute of Mathematics University of Tsukuba Ibaragi 305, Japan 